\title{
ON THE STRUCTURE OF THE SOLUTION SET OF ABSTRACT INCLUSIONS WITH INFINITE DELAY IN A BANACH SPACE
}

\author{
LAHCENE GUEDDA
}

\begin{abstract}
In this paper we study the topological structure of the solution set of abstract inclusions, not necessarily linear, with infinite delay on a Banach space defined axiomatically. By using the techniques of the theory of condensing maps and multivalued analysis tools, we prove that the solution set is a compact $R_{\delta}$-set. Our approach makes possible to give a unified scheme in the investigation of the structure of the solution set of certain classes of differential inclusions with infinite delay.
\end{abstract}

\section{Introduction}

When an existence result is proved for the Cauchy problem for a class of systems where the solutions are not unique, it is natural to discuss for this class the topological structure of the solution set. For this reason in recent years much work has been done in that direction. It was Aronszajn [2], who first proved that the solution set of the Cauchy problem $x^{\prime}(t)=f(t, x(t))$ for almost every $t \in[0, T], x(0)=x_{0}$, where $f(\cdot, \cdot)$ is a bounded, continuous function on $[0, T] \times \mathbb{R}^{n}$, is an $R_{\delta}$-set. This result was extended to differential inclusions by Himmelberg-Van Vleck [22] and De Blasi-Myjak [12] for differential inclusions in $\mathbb{R}^{n}$ and by Bothe [5], M. Cichoń-Kubiaczyk [8], Deimling-Rao [14],

2010 Mathematics Subject Classification. Primary: 34K30, 47H08; Secondary: 34K09, 93C20.

Key words and phrases. Abstract inclusion; functional differential inclusion; infinite delay; measures of noncompactness and condensing mappings; $R_{\delta}$-set.

The author wishes to thank referees for their insightful comments and helpful suggestions. 
Conti-Obukhovski1-Zecca [10], and Papageorgiou [27] for differential inclusions in Banach spaces. For more literature and some recent developments on the topic see [16], [20] and the references cited therein.

In [17], existence and continuous dependence results are presented, and the $R_{\delta}$-structure of the solution set is claimed for a semilinear differential inclusion with infinite delay in a Banach space.

We aim in this paper to study the topological structure of the solution set of abstract inclusions, not necessarily linear, with infinite delay in a Banach space.

More precisely, let $\sigma$ be a real number and $T>0$ be a fixed time. By $C([\sigma, T+\sigma] ; E)$ we denote the space of continuous functions defined on $[\sigma, T+\sigma]$ with values in a Banach space $(E,\|\cdot\|)$, endowed with the uniform convergence norm and by $L^{1}([\sigma, T+\sigma] ; E)$ we denote the space of all Bochner summable functions endowed with the usual norm. For any function $z:(-\infty, \sigma+T] \rightarrow E$ and for every $t \in[\sigma, \sigma+T], z_{t}$ represents the function from $(-\infty, 0]$ into $E$ defined by $z_{t}(\theta)=z(t+\theta) ;-\infty<\theta \leq 0$. Let $\mathcal{B}$ be a Banach space of functions mapping $(-\infty ; 0]$ into $E$ endowed with a norm $\|\cdot\|_{\mathcal{B}}$ and satisfying the following axioms:

If $z:(-\infty, \sigma+T] \rightarrow E$ is continuous on $[\sigma, \sigma+T]$ and $z_{\sigma} \in \mathcal{B}$, then, for every $t \in[\sigma, \sigma+T]$, we have

(B1) $z_{t} \in \mathcal{B}$;

(B2) $\left\|z_{t}\right\|_{\mathcal{B}} \leq K(t-\sigma) \sup _{\sigma \leq s \leq t}\|z(s)\|+N(t-\sigma)\left\|z_{\sigma}\right\|_{\mathcal{B}}$, where $K, N:[0,+\infty) \rightarrow$ $[0,+\infty)$ are independent of $z, K$ is positive and continuous, and $N$ is locally bounded;

(B3) the function $t \mapsto z_{t}$ is continuous;

(B4) $\|z(t)\|_{E} \leq l\left\|z_{t}\right\|_{\mathcal{B}}$, where $l>0$ is a constant independent of $z$.

A space satisfying (B1)-(B4) was first introduced by Hale and Kato [21] and has been considered as a phase space in the theory of retarded functional equations (see [9], [26], [28]). Let us give two examples of Banach spaces $\mathcal{B}$ satisfying axioms (B1)-(B4), see for example [23] and [24, p. 20].

Let $g(\theta), \theta \in(-\infty, 0]$, be a positive continuous function such that $g(\theta) \rightarrow \infty$ as $\theta \rightarrow-\infty$.

The space $U C_{g}$. The space $U C_{g}$ is a set of continuous functions $\phi$ such that $\phi / g$ is bounded and uniformly continuous in $(-\infty, 0]$. Set

$$
\|\phi\|_{\mathcal{B}}=\sup \{\|\phi(\theta)\| / g(\theta): \theta \in(-\infty, 0]\} .
$$

$U C_{g}$ is a Banach space satisfying axioms (B1)-(B4). 
The space $\mathcal{L}_{g}$. The space $\mathcal{L}_{g}$ is the set of strongly measurable functions $\phi$ such that $\|\phi\| / g$ is integrable over $(-\infty, 0]$. Set

$$
\|\phi\|_{\mathcal{B}}=\|\phi(0)\|+\int_{-\infty}^{0}\|\phi(\theta)\| / g(\theta) d \theta .
$$

$\mathcal{L}_{g}$ is a Banach space satisfying axioms (B1)-(B4).

Let us denote by the symbol $\mathcal{C}((-\infty, \sigma+T] ; E)$ the space consisting of functions $z:(-\infty, T+\sigma] \rightarrow E$ such that $z_{\sigma} \in \mathcal{B}$ and the restriction $\left.z\right|_{[\sigma, T+\sigma]}$ is continuous, endowed with the norm

$$
\|z\|_{\mathcal{C}}=\left\|z_{\sigma}\right\|_{\mathcal{B}}+\left\|\left.z\right|_{[\sigma, T+\sigma]}\right\|_{C([\sigma, T+\sigma] ; E)} .
$$

It is easy to see that $\mathcal{C}((-\infty, \sigma+T] ; E)$ endowed with the norm $\|\cdot\|_{\mathcal{C}}$ is a Banach space.

Let us consider abstract inclusions with infinite delay on $\mathcal{B}$ described in the form

$$
\left\{\begin{array}{l}
z \in S \circ \operatorname{sel}_{F}(z) \quad \text { on }[\sigma, \sigma+T], \\
z_{\sigma}=\varphi
\end{array}\right.
$$

where $\varphi \in \mathcal{B}, F:[\sigma, T+\sigma] \times \mathcal{B} \rightarrow 2^{E}$ is a compact convex valued multimap satisfying upper Carathéodory and $\chi$ regularity conditions, where $\chi$ denotes the Hausdorff measure of noncompactness in $E$, the symbol sel $F$ denotes the superposition operator generated by $F$, i.e. for any $z \in \mathcal{C}((-\infty, \sigma+T] ; E)$,

$$
\operatorname{sel}_{F}(z)=\left\{f \in L^{1}([\sigma, T+\sigma] ; E): f(s) \in F\left(s, z_{s}\right) \text { for a.e. } s \in[\sigma, T+\sigma]\right\},
$$

finally, $S: L^{1}([\sigma, T+\sigma] ; E) \rightarrow C([\sigma, T+\sigma] ; E)$ is an abstract operator satisfying $S(f)(\sigma)=\varphi(0)$ for all $f \in L^{1}([\sigma, T+\sigma] ; E)$. By $S \circ \operatorname{sel}_{F}(z)$ we mean the set

$$
\left\{S(f)(\cdot) ; f \in \operatorname{sel}_{F}(z)\right\} .
$$

Definition 1.1. A function $z \in \mathcal{C}((-\infty, \sigma+T] ; E)$ is a solution of (1.1) if $z_{\sigma}=\varphi$ and there exists $f \in \operatorname{sel}_{F}(z)$ such that $\left.z\right|_{[\sigma, T+\sigma]}=S(f)$.

Definition 1.2. For $z \in \mathcal{C}((-\infty, \sigma+T] ; E)$ with $z_{\sigma}=\varphi$, if there exist $b$, $\sigma<b \leq T$, and $f \in \operatorname{sel}_{F}(z)$ such that $\left.z\right|_{[\sigma, b+\sigma]}=\left.S(f)\right|_{[\sigma, b+\sigma]}$, then we say that $\widehat{z}=\left.z\right|_{(-\infty, b+\sigma]}$ is a local solution of (1.1).

We aim in this paper to give an answer to the following problem:

Suppose that the solution set of inclusion (1.1) is nonempty and bounded in $\mathcal{C}((-\infty, \sigma+T] ; E)$ and suppose that each local solution has an extension to all $(-\infty, \sigma+T]$. Under which conditions on the operator $S$ this set is a compact $R_{\delta}$-set in $\mathcal{C}((-\infty, \sigma+T] ; E)$ ?

Giving an answer to this problem it makes possible to present a unified scheme in the investigation of the structure of the solution set for certain classes 
of differential inclusions with infinite delay and to generalize and improve all theorems of Aronszajn-type to such classes of differential inclusions without or with finite delay, as shows the following illustration:

Consider the Cauchy problem for differential inclusions with infinite delay of the form

$$
\left\{\begin{array}{l}
z^{\prime}(t) \in A(t, z(t))+F\left(t, z_{t}\right) \quad \text { for } t \in[\sigma, T+\sigma], \\
z_{\sigma}=\varphi .
\end{array}\right.
$$

In this situation as an operator $S$ we take the solution operator of the quasi-linear problem

$$
\left\{\begin{array}{l}
z^{\prime}(t) \in A(t, z(t))+f(t) \quad \text { for } t \in[\sigma, T+\sigma], \\
z(\sigma)=\varphi(0),
\end{array}\right.
$$

i.e., for every $f \in L^{1}([\sigma, T+\sigma] ; E)$, the value $S(f)$ stands for the (unique) solution of problem (1.3). Then, problem (1.2) can be written as

$$
\left\{\begin{array}{l}
y \in S \circ \operatorname{sel}_{F}(y) \\
y_{\sigma}=\varphi
\end{array}\right.
$$

Finally, let us mention that after a convenient formulation of problem (1.1), our presented scheme of the proof in this paper is inspired from [5], [8], [10] and [25, Theorem 5.3.1].

\section{Preliminaries}

Let us recall some notations (see, e.g. [1], [13], [19], [25]). A Hausdorff topological space $\Lambda$ is said to be contractible if the identity map $i_{\Lambda}: \Lambda \rightarrow \Lambda$ is null homotopic, i.e. there exist a continuous $h:[0,1] \times \Lambda \rightarrow \Lambda$ and $x_{0} \in \Lambda$ such that $h(0, x)=x$ and $h(1, x)=x_{0}$ for all $x \in \Lambda$. A subset of a metric space is an $R_{\delta}$-set if it is the intersection of a decreasing sequence of nonempty compact contractible sets.

Let $X, Y$ be two topological vector spaces. We denote by $\mathcal{P}(Y)$ the family of all nonempty subsets of $Y$ and by $K(Y)$ (resp. $K v(Y)$ ), we denote the collection of all nonempty compact (resp. nonempty compact convex) subsets of $Y$.

A multivalued map $F: X \rightarrow \mathcal{P}(Y)$ is said to be:

(i) upper semicontinuous (u.s.c.), if $F^{-1}(O)=\{x \in X: F(x) \subset O\}$ is an open subset of $X$ for every open $O \subset Y$;

(ii) lower semicontinuous (1.s.c.), if $F^{-1}(Q)=\{x \in X: F(x) \subset Q\}$ is a closed subset of $X$ for every closed $Q \subset Y$;

(iii) continuous, if it is both upper and lower semicontinuous;

(iv) closed, if its graph $\Gamma_{F}=\{(x, y) \in X \times Y: y \in F(x)\}$ is a closed subset of $X \times Y$. 
From [25, Example 1.1.6, Theorem 1.1.7] one can easily show the following simple result:

LEMmA 2.1. If $Y$ is a Banach space and $\mathcal{M} \subset C([a, b] ; Y)$ is a compact set, then the map

$$
\mathcal{M}(\cdot):[a, b] \rightarrow K(Y), \quad t \mapsto \mathcal{M}(t)
$$

is continuous and the set $\mathcal{M}([a, b]) \subset Y$ is compact.

Let $E$ be a Banach space and $(\mathcal{Y}, \leq)$ be a partially ordered set. A map $\Psi: \mathcal{P}(E) \rightarrow \mathcal{Y}$ is called a measure of noncompactness in $E$ if $\Psi(\Omega)=\Psi(\overline{\mathrm{co}} \Omega)$ for every $\Omega \subset \mathcal{P}(E)$, where $\overline{\mathrm{co}} \Omega$ denotes the closed convex hull of $\Omega$. The measure of noncompactness $\Psi$ is called:

(i) nonsingular, if for every $a \in E, \Omega \in \mathcal{P}(E), \Psi(\{a\} \cup \Omega)=\Psi(\Omega)$;

(ii) monotone, if $\Omega_{0}, \Omega_{1} \in \mathcal{P}(E)$ and $\Omega_{0} \subseteq \Omega_{1}$ imply $\Psi\left(\Omega_{0}\right) \leq \Psi\left(\Omega_{1}\right)$.

If $\mathcal{Y}$ is a cone in a Banach space we will say that

(iii) $\Psi$ is regular, if $\Psi(\Omega)=0$ is equivalent to the relative compactness of $\Omega$.

One of most important examples of a measure of noncompactness possessing all these properties is the Hausdorff measure of noncompactness, defined by

$$
\chi(\Omega)=\inf \{\varepsilon>0: \Omega \text { has a finite } \varepsilon \text {-net in } E\} .
$$

The sequential measure of noncompactness $\tilde{\chi}: \mathcal{P}(E) \rightarrow \mathbb{R}^{+}$generated by $\chi$ is given by

$$
\widetilde{\chi}(\Omega)=\sup _{\mathcal{D} \in \Delta(\Omega)} \chi(\mathcal{D}),
$$

where $\Delta(\Omega)$ is the collection of all denumerable subsets of $\Omega$.

Lemma 2.2. We have $\widetilde{\chi}(\Omega) \leq \chi(\Omega) \leq 2 \widetilde{\chi}(\Omega)$.

Let $\Psi$ be a measure of noncompactness in $E$. A multimap $G: Z \rightarrow K(E)$, where $Z \subset E$ is a closed subset, is called $\Psi$-condensing if for every bounded set $\Omega \subset Z$, the relation $\Psi(G(\Omega)) \geq \Psi(\Omega)$ implies the relative compactness of $\Omega$. For more details, see for example $[3,25]$.

By the symbol $L^{1}([a, b] ; E)$ we denote the space of all Bochner summable functions.

A multifunction $\digamma:[a, b] \rightarrow K(E)$ is said to be:

(i) strongly measurable, if there exists a sequence $\left\{\digamma_{n}\right\}_{n=1}^{\infty}$ of step multifunctions such that $\operatorname{Haus}\left(\digamma(t)-\digamma_{n}(t)\right) \rightarrow 0$ as $n \rightarrow \infty$ for $\mu$-a.e. $t \in[a, b]$, where $\mu$ denotes a Lebesgue measure on $[a, b]$ and Haus denotes the Hausdorff metric on $K(E)$. Every strongly measurable multivalued map $\digamma$ admits a strongly measurable selection, i.e. there exists $f:[a, b] \rightarrow E$ measurable with $f(t) \in \digamma(t)$ for almost every $t \in[a, b]$;

(ii) integrable, if it has a Bochner summable selection $f \in L^{1}([a, b] ; E)$; 
(iii) integrably bounded, if in $L^{1}\left([a, b] ; \mathbb{R}^{+}\right)$there exists a summable function $q(\cdot)$ such that $\|\digamma(t)\|=\sup \{\|y\|: y \in \digamma(t)\} \leq q(t)$ for almost every $t \in[a, b]$.

For more details, see for example [13], [19], [25].

A sequence $\left\{f_{n}\right\}_{n=1}^{\infty} \subset L^{1}([a, b] ; E)$ is semicompact if:

(i) it is integrably bounded: $\left\|f_{n}(t)\right\| \leq q(t)$ for almost every $t \in[a, b]$ and for every $n \geq 1$, where $q(\cdot) \in L^{1}\left([a, b] ; \mathbb{R}^{+}\right)$;

(ii) the set $\left\{f_{n}(t)\right\}_{n=1}^{\infty}$ is relatively compact for almost every $t \in[a, b]$.

Lemma $2.3([15])$. Any semicompact sequence in $L^{1}([a, b] ; E)$ is weakly compact in $L^{1}([a, b] ; E)$.

\section{Formulation of the problem, statement of the result}

Throughout this paper $\mathcal{B}$ is considered as a Banach space of functions mapping $(-\infty ; 0]$ into $E$ endowed with a norm $\|\cdot\|_{\mathcal{B}}$ and satisfying axioms (B1)-(B4). Let $\sigma$ be a real number, $T>0$ a fixed time and $E$ an arbitrary Banach space. We denote by $\Sigma_{\varphi}^{F} \subset \mathcal{C}((-\infty, \sigma+T] ; E)$ the solution set of (1.1).

3.1. Hypotheses. We impose the following hypotheses.

The map $S: L^{1}([\sigma, T+\sigma] ; E) \rightarrow C([\sigma, T+\sigma] ; E)$ satisfies the following conditions:

$\left(\mathrm{S}_{0}\right)$ for all $f \in L^{1}([\sigma, T+\sigma] ; E), S(f)(\sigma)=\varphi(0)$;

$\left(\mathrm{S}_{1}\right)$ there exists $M>0$ such that for all $f, g \in L^{1}([\sigma, T+\sigma] ; E)$,

$$
\|S(f)(t)-S(g)(t)\| \leq M\|S(f)(s)-S(g)(s)\|+M \int_{s}^{t}\|f(\gamma)-g(\gamma)\| d \gamma,
$$

for $\sigma \leq s \leq t \leq T+\sigma$;

$\left(\mathrm{S}_{2}\right)$ for any compact $K \subset E$ and sequence $\left\{f_{n}\right\}_{n=1}^{\infty} \subset L^{1}([\sigma, T+\sigma] ; E)$ such that $\left\{f_{n}(t)\right\}_{n=1}^{\infty} \subset K$ for almost every $t \in[\sigma, T+\sigma]$, the weak convergence $f_{0} \underset{w}{\rightarrow} f_{n}$ implies $S\left(f_{n}\right) \rightarrow S f_{0}$ in $C([\sigma, T+\sigma] ; E)$.

The multimap $F:[\sigma, \sigma+T] \times \mathcal{B} \rightarrow K v(E)$ satisfies the following conditions:

$\left(\mathrm{F}_{1}\right)$ the multimap $F:(\cdot, u) \rightarrow K v(E)$ has a strongly measurable selector for every $u \in \mathcal{B}$;

$\left(\mathrm{F}_{2}\right)$ the multimap $F:(t, \cdot) \rightarrow K v(E)$ is u.s.c. for almost every $t \in[\sigma, \sigma+T]$;

$\left(\mathrm{F}_{3}\right)$ there exists a function $\alpha \in L^{1}\left([\sigma, \sigma+T] ; \mathbb{R}^{+}\right)$such that for every $u \in \mathcal{B}$,

$$
\|F(t, u)\| \leq \alpha(t)(1+\|u\|) \quad \text { a.e. } t \in[\sigma, \sigma+T]
$$

$\left(\mathrm{F}_{4}\right)$ there exists a function $\kappa(\cdot) \in L^{1}\left([\sigma, \sigma+T] ; \mathbb{R}^{+}\right)$such that for every bounded $\Omega \subset \mathcal{B}$,

$$
\chi(F(t, \Omega)) \leq \kappa(t) \sup _{-\infty<\theta \leq 0} \chi(\Omega(\theta)),
$$

for almost every $t \in[\sigma, \sigma+T]$, where $\Omega(\theta)=\{u(\theta): u \in \Omega\}$. 
3.2. Main result. We can now state the main result of this paper.

ThEOREM 3.1. Suppose that under conditions $\left(\mathrm{S}_{0}\right)-\left(\mathrm{S}_{2}\right)$ and $\left(\mathrm{F}_{1}\right)-\left(\mathrm{F}_{4}\right)$, the following are valid:

$\left(\mathcal{I}_{1}\right)$ the set $\Sigma_{\varphi}^{F}$ is nonempty and bounded;

$\left(\mathcal{I}_{2}\right)$ each local solution of $(1.1)$ has an extension to all $(-\infty, T+\sigma]$.

Then $\Sigma_{\varphi}^{F}$ is a compact $R_{\delta}$-subset of $\mathcal{C}((-\infty, \sigma+T] ; E)$.

REMARK 3.2. Note that if the set $\Sigma_{\varphi}^{F}$ is nonempty then it is necessarily bounded thanks to $\left(\mathrm{F}_{3}\right)$ and $\left(\mathrm{S}_{0}\right)-\left(\mathrm{S}_{1}\right)$. Indeed, let $x \in \Sigma_{\varphi}^{F}$. Then $x_{\sigma}=\varphi$ and $x(\cdot)=S(f)(\cdot)$ on $[\sigma, T+\sigma]$ for some $f \in \operatorname{sel}_{F}(x)$. By using axiom (B3) and conditions $\left(\mathrm{S}_{0}\right),\left(\mathrm{S}_{1}\right)$ and $\left(\mathrm{F}_{3}\right)$, for any $t \in[\sigma, T+\sigma]$, we have the following estimate:

$$
\begin{aligned}
\|x(t)\| & =\|S(f)(t)\| \leq \max _{t \in[\sigma, T+\sigma]}\|S(0)(t)\|+M \int_{\sigma}^{t}\|f(s)\| d s \\
& \leq \max _{t \in[\sigma, T+\sigma]}\|S(0)(t)\|+M \int_{\sigma}^{t} \alpha(s)\left(1+\left\|x_{s}\right\|\right) d s \\
& \leq \omega_{2}+\omega_{1} \int_{\sigma}^{t} \alpha(s) \max _{\theta \in[\sigma, s]}\|x(\theta)\| d s
\end{aligned}
$$

where

$$
\begin{aligned}
& \omega_{1}=M \max _{t \in[\sigma, T+\sigma]} K(t-\sigma) ; \\
& \omega_{2}=\max _{t \in[\sigma, T+\sigma]}\|S(0)(t)\|+M\|\alpha\|_{L^{1}}\left(1+\|\varphi\|_{\mathcal{B}} \sup _{t \in[\sigma, T+\sigma]} N(t-\sigma)\right) .
\end{aligned}
$$

Since the last expression does not decrease, we have

$$
\max _{s \in[\sigma, t]}\|x(s)\| \leq \omega_{2}+\omega_{1} \int_{\sigma}^{t} \alpha(s) \max _{\theta \in[\sigma, s]}\|x(\theta)\| d s .
$$

Applying the Gronwall-Bellmann inequality to the function $t \mapsto \max _{s \in[\sigma, t]}\|x(s)\|$, $t \in[\sigma, T+\sigma]$, we obtain

$$
\max _{s \in[\sigma, t]}\|x(s)\| \leq \omega_{2} \exp \left(\omega_{1} \int_{\sigma}^{t} \alpha(s) d s\right) .
$$

Therefore

$$
\|x\|_{\mathcal{C}} \leq\|\varphi\|_{\mathcal{B}}+\omega_{2} \exp \left(\omega_{1} \int_{\sigma}^{T+\sigma} \alpha(s) d s\right) .
$$

As a consequence, for $\left(\mathcal{I}_{1}\right)$ one can assume that $\sum_{\varphi}^{F}$ is only nonempty.

REMARK 3.3. In Section 4, we describe some concrete situations where the conditions of Theorem 3.1 are verified. 
Before giving the proof of Theorem 3.1, we need some auxiliary notation and we prove some auxiliary results.

In the space $C([\sigma, T+\sigma], E)$, let us define the set

$$
D(\varphi, T)=\{x \in C([\sigma, T+\sigma] ; E]): x(\sigma)=\varphi(0)\} .
$$

It is clear that $D(\varphi, T)$ is a closed convex subset in $C([\sigma, T+\sigma] ; E)$.

For every $x \in D(\varphi, T)$, let us define the function $x[\varphi] \in C((-\infty, T+\sigma] ; E)$ by

$$
x[\varphi](t)= \begin{cases}\varphi(t-\sigma) & \text { if }-\infty<t<\sigma, \\ x(t) & \text { if } \sigma \leq t \leq T+\sigma .\end{cases}
$$

Then

$$
x[\varphi]_{t}(\theta)= \begin{cases}\varphi(t-\sigma+\theta) & \text { if }-\infty<\theta<\sigma-t, \\ x(t+\theta) & \text { if } \sigma-t \leq \theta \leq 0 .\end{cases}
$$

The function $\left.x[\varphi]\right|_{[\sigma, T+\sigma]}=x(\cdot)$ is continuous and $x[\varphi]_{\sigma}=\varphi$, hence by axiom (B1), $x[\varphi]_{t} \in \mathcal{B}$ for all $t \in[\sigma, T+\sigma]$.

Let the map $\varrho:[\sigma, T+\sigma] \times D(\varphi, T) \rightarrow \mathcal{B}$ be defined by

$$
\varrho(t, x)=x[\varphi]_{t} .
$$

It is known (see [18, Section 3]) that, for every $x \in D(\varphi, T)$, the map $\varrho(\cdot, x):[\sigma$, $T+\sigma] \rightarrow \mathcal{B}$ is continuous and the map $\varrho(t, \cdot): D(\varphi, T) \rightarrow \mathcal{B}$ is Lipschitz continuous uniformly with respect to $t \in[\sigma, T+\sigma]$.

Let us consider the superposition operator $\overline{\operatorname{sel}}_{F}: D(\varphi, T) \rightarrow \mathcal{P}\left(L^{1}([\sigma, T+\right.$ $\sigma] ; E)$ ), defined as

$$
\begin{aligned}
& \overline{\operatorname{sel}}_{F}(x)=\operatorname{sel}_{F}(x[\varphi])=\left\{f \in L^{1}([\sigma, T+\sigma] ; E):\right. \\
& \left.\qquad f(s) \in F(s, \varrho(s, x))=F\left(s, x[\varphi]_{s}\right) \text { for a.e. } s \in[\sigma, T+\sigma]\right\} .
\end{aligned}
$$

The superposition operator $\overline{\operatorname{sel}}_{F}$ is well-defined (see [18, Remark 3]). Moreover, using the uniform Lipschitz continuity of $\varrho(t, \cdot)$ and [25, Lemma 5.1.1], we obtain the following property of weak closeness of $\overline{\operatorname{sel}}_{F}$ (see also [18, Lemma 4]).

LEMmA 3.4. If the sequences $\left\{x^{n}\right\}_{n=1}^{\infty} \subset D(\varphi, T),\left\{f_{n}\right\}_{n=1}^{\infty} \subset L^{1}([\sigma, T+\sigma], E)$ with $f_{n} \in \overline{\operatorname{sel}}_{F}\left(x^{n}\right), n \geq 1$, are such that $x^{n} \rightarrow x^{0}, f_{n} \stackrel{\mathrm{w}}{\longrightarrow} f_{0}$, then $f_{0} \in \overline{\operatorname{sel}}_{F}\left(x^{0}\right)$.

In $D(\varphi, T)$, let us consider the following problem:

$$
x \in S \circ \overline{\operatorname{sel}}_{F}(x) .
$$

Denote by $\Sigma_{\varphi(0)}^{F} \subset D(\varphi, T)$ the solution set of (3.4).

LemmA 3.5. We have $\Sigma_{\varphi}^{F}=\left\{x[\varphi]: x \in \Sigma_{\varphi(0)}^{F}\right\}$ and $\Sigma_{\varphi(0)}^{F}=\left.\Sigma_{\varphi}^{F}\right|_{[\sigma, \sigma+T]}$. 
Proof. It is enough to observe that by condition $\left(\mathrm{S}_{0}\right)$, the mutimap $S \circ$ $\overline{\operatorname{sel}}_{F}(\cdot)$ maps $D(\varphi, T)$ into itself and

$$
\Sigma_{\varphi(0)}^{F}=\operatorname{Fix}\left(S \circ \overline{\operatorname{sel}}_{F}\right)=\left.\operatorname{Fix}\left(S \circ \operatorname{sel}_{F}\right)\right|_{[\sigma, \sigma+T]}=\left.\Sigma_{\varphi}^{F}\right|_{[\sigma, \sigma+T]} .
$$

Corollary 3.6. From conditions $\left(\mathcal{I}_{1}\right)-\left(\mathcal{I}_{2}\right)$ of Theorem 3.1 , it results that the set $\Sigma_{\varphi(0)}^{F}$ is nonempty bounded. Moreover, each local solution of (3.4) has an extension to all $[\sigma, T+\sigma]$, i.e. if $x \in D(\varphi, T)$ satisfies $\left.x\right|_{[\sigma, b+\sigma]} \in S$ 。 $\left.\overline{\operatorname{sel}}_{F}(x)\right|_{[\sigma, b+\sigma]}$, for some $\sigma<b \leq T$, then $x$ has an extension to all $[\sigma, T+\sigma]$.

The scheme of our proof of Theorem 3.1 is as follows: first, we prove that $\Sigma_{\varphi(0)}^{F}$ is a compact $R_{\delta}$-set in $D(\varphi, T)$, then, by using a simple result (see Lemma 3.23), we deduce that $\Sigma_{\varphi}^{F}$ is a compact $R_{\delta}$-set in $\mathcal{C}((-\infty, \sigma+T] ; E)$.

The next two lemmas give some properties of the operator $S$ which will be needed in the sequel.

LEMмA 3.7. If $S$ satisfies conditions $\left(\mathrm{S}_{0}\right)$ and $\left(\mathrm{S}_{1}\right)$, then $S$ satisfies

$\left(1 \mathrm{~S}_{1}\right)$ for all $f, g \in L^{1}([\sigma, T+\sigma] ; E)$,

$$
\|S(f)(t)-S(g)(t)\| \leq M \int_{\sigma}^{t}\|f(\theta)-g(\theta)\| d \theta, \quad \sigma \leq t \leq T+\sigma ;
$$

$\left(2 \mathrm{~S}_{1}\right)$ for all $g_{0}, g_{1}, g_{2} \in L^{1}([\sigma, T+\sigma], E)$ if $S g_{1}=S g_{2}$, then

$$
S\left(\mathbf{1}_{[\sigma, \theta]} g_{1}+\mathbf{1}_{[\theta, T+\sigma]} g_{0}\right)=S\left(\mathbf{1}_{[\sigma, \theta]} g_{2}+\mathbf{1}_{[\theta, T+\sigma]} g_{0}\right),
$$

for all $\theta \in[\sigma, T+\sigma]$, where $\mathbf{1}_{[a, b]}$ denotes the characteristic function of the interval $[a, b]$.

Proof. Taking $s=\sigma$ in $\left(\mathrm{S}_{1}\right)$ and using $\left(\mathrm{S}_{0}\right)$, we deduce $\left(1 \mathrm{~S}_{1}\right)$. It remains to show $\left(2 \mathrm{~S}_{1}\right)$. Let $\theta \in[\sigma, T+\sigma]$. Remark that from $\left(\mathrm{S}_{1}\right)$ it follows immediately that

$$
S\left(\mathbf{1}_{[\sigma, \theta]} g_{1}+\mathbf{1}_{[\theta, T+\sigma]} g_{0}\right)(t)=S\left(g_{1}\right)(t) \quad \text { for all } t \in[\sigma, \theta] .
$$

Indeed, for $t \in[\sigma, \theta]$, using $\left(\mathrm{S}_{1}\right)$ and $\left(\mathrm{S}_{0}\right)$, we have

$$
\begin{aligned}
\| S\left(\mathbf{1}_{[\sigma, \theta]} g_{1}\right. & \left.+\mathbf{1}_{[\theta, T+\sigma]} g_{0}\right)(t)-S\left(g_{1}\right)(t) \| \\
\leq & M\left\|S\left(\mathbf{1}_{[\sigma, \theta]} g_{1}+\mathbf{1}_{[\theta, T+\sigma]} g_{0}\right)(\sigma)-S\left(g_{1}\right)(\sigma)\right\| \\
& \quad+M \int_{\sigma}^{t}\left\|\mathbf{1}_{[\sigma, \theta]}(\tau) g_{1}(\tau)+\mathbf{1}_{[\theta, T+\sigma]}(\tau) g_{0}(\tau)-g_{1}(\tau)\right\| d \tau \\
\leq & M\|\varphi(0)-\varphi(0)\|+\int_{\sigma}^{t}\left\|g_{1}(\tau)-g_{1}(\tau)\right\| d \tau=0 .
\end{aligned}
$$

By symmetry, we also have

$$
S\left(\mathbf{1}_{[\sigma, \theta]} g_{2}+\mathbf{1}_{[\theta, T+\sigma]} g_{0}\right)(t)=S\left(g_{2}\right)(t) \quad \text { for all } t \in[\sigma, \theta] .
$$


Since $S\left(g_{1}\right)=S\left(g_{2}\right)$, from (3.6) and (3.7), it results that for all $t \in[\sigma, \theta]$,

$$
S\left(\mathbf{1}_{[\sigma, \theta]} g_{1}+\mathbf{1}_{[\theta, T+\sigma]} g_{0}\right)(t)=S\left(\mathbf{1}_{[\sigma, \theta]} g_{2}+\mathbf{1}_{[\theta, T+\sigma]} g_{0}\right)(t) .
$$

Now, for $t \in[\theta, T+\sigma]$, using again $\left(\mathrm{S}_{1}\right)$ and bearing in mind (3.8), we have

$$
\begin{aligned}
& \left\|S\left(\mathbf{1}_{[\sigma, \theta]} g_{1}+\mathbf{1}_{[\theta, T+\sigma]} g_{0}\right)(t)-S\left(\mathbf{1}_{[\sigma, \theta]} g_{2}+\mathbf{1}_{[\theta, T+\sigma]} g_{0}\right)(t)\right\| \\
& \leq\left\|S t\left(\mathbf{1}_{[\sigma, \theta]} g_{1}+\mathbf{1}_{[\theta, T+\sigma]} g_{0}\right)(\theta)-S\left(\mathbf{1}_{[\sigma, \theta]} g_{2}+\mathbf{1}_{[\theta, T+\sigma]} g_{0}\right)(\theta)\right\| \\
& +\int_{\theta}^{t}\left\|\mathbf{1}_{[\sigma, \theta]}(\tau) g_{1}(\tau)+\mathbf{1}_{[\theta, T+\sigma]}(\tau) g_{0}(\tau)-\mathbf{1}_{[\sigma, \theta]}(\tau) g_{2}(\tau)-\mathbf{1}_{[\theta, T+\sigma]}(\tau) g_{0}(\tau)\right\| d \tau=0 .
\end{aligned}
$$

Hence, for all $t \in[\theta, T+\sigma]$,

$$
S\left(\mathbf{1}_{[\sigma, \theta]} g_{1}+\mathbf{1}_{[\theta, T+\sigma]} g_{0}\right)(t)=S\left(\mathbf{1}_{[\sigma, \theta]} g_{2}+\mathbf{1}_{[\theta, T+\sigma]} g_{0}\right)(t) .
$$

Condition $\left(2 \mathrm{~S}_{1}\right)$ follows from equalities (3.8) and (3.9).

Lemma 3.8. Assume that the operator $S$ satisfies conditions $\left(1 \mathrm{~S}_{1}\right)$ and $\left(\mathrm{S}_{2}\right)$. Then:

(a) if the sequence of functions $\left\{f_{n}\right\}_{n=1}^{\infty} \subset L^{1}([a, b] ; E)$ is such that

$$
\begin{aligned}
& \qquad\left\|f_{n}(t)\right\| \leq \delta(t), \quad \text { for all } n=1,2, \ldots \text { and a.e. } t \in[\sigma, T+\sigma] \\
& \text { and } \chi\left(\left\{f_{n}\right\}_{n=1}^{\infty}\right) \leq \zeta(t) \text { for a.e. } t \in[a, b] \text {, where } \delta, \zeta \in L_{+}^{1}([\sigma, T+\sigma]) \text {, } \\
& \text { then } \\
& \qquad \chi\left(S\left\{f_{n}(t)\right\}_{n=1}^{\infty}\right) \leq 2 M \int_{\sigma}^{t} \zeta(s) d s, \quad \text { for all } t \in[\sigma, T+\sigma] ;
\end{aligned}
$$

(b) for every semicomapct sequence $\left\{f_{n}\right\}_{n=1}^{\infty} \subset L^{1}([\sigma, T+\sigma]$; E) the sequence $\left\{S\left(f_{n}\right)\right\}_{n=1}^{\infty}$ is relatively compact in $D(\varphi, T)$, and; moreover, if $f_{n} \stackrel{\mathrm{w}}{\longrightarrow}$ $f_{0}$ then $S\left(f_{n}\right) \rightarrow S f_{0}$.

Proof. See, for example, [25, Theorems 4.2.2, 5.1.1].

Using standard arguments, we have:

Lemma 3.9. We can assume without loss of generality that $F$ satisfies the following global estimate:

$\left(\mathrm{F}_{3}^{\prime}\right)$ there exists a function $\bar{\alpha} \in L^{1}\left([\sigma, \sigma+T] ; \mathbb{R}^{+}\right)$such that for every $u \in \mathcal{B}$,

$$
\|F(t, u)\| \leq \bar{\alpha}(t), \quad \text { for a.e. } t \in[\sigma, \sigma+T] .
$$

Proof. Let $p>0$ be chosen such that $\left\|\Sigma_{\varphi(0)}^{F}\right\|_{C([\sigma, T+\sigma] ; E)} \leq p$. Note that the existence of $p$ is ensured by Corollary 3.6. Set

$$
\begin{aligned}
& \beta_{K}=\max _{t \in[0, T]} K(t) ; \\
& \beta_{N}=\sup _{t \in[0, T]} N(t) .
\end{aligned}
$$


By axiom (B2), we obtain, for all $x \in \Sigma_{\varphi(0)}^{F}$,

$$
\left\|x[\varphi]_{t}\right\| \leq \beta_{K} \sup _{s \in[\sigma, t+\sigma]}\|x(s)\|+\beta_{N}\|\varphi\|_{\mathcal{B}} \leq p \beta_{K}+\beta_{N}\|\varphi\|_{\mathcal{B}} .
$$

Set $\widetilde{p}=p \beta_{K}+\beta_{N}\|\varphi\|_{\mathcal{B}}$. Let $\bar{F}:[\sigma, T+\sigma] \times \mathcal{B} \rightarrow K v(E)$ be a multimap defined as

$$
\bar{F}(t, u)=F\left(t, p_{r}(u)\right),
$$

where $p_{r}: \mathcal{B} \rightarrow B_{\mathcal{B}}(0, \widetilde{p})$ is the radial projection, i.e.

$$
p_{r}(u)= \begin{cases}u & \text { if }\|u\| \leq \widetilde{p}, \\ \widetilde{p} u /\|u\| & \text { if }\|u\|>\widetilde{p} .\end{cases}
$$

Since $p_{r}$ is a Lipschitz map, it is easily seen that $\bar{F}$ satisfies conditions $\left(\mathrm{F}_{1}\right)$, $\left(\mathrm{F}_{2}\right),\left(\mathrm{F}_{4}\right)$, condition $\left(\mathrm{F}_{3}^{\prime}\right)$ with $\bar{\alpha}(t)=\alpha(t)(1+\widetilde{p})$, and $\Sigma_{\varphi(0)}^{F}=\Sigma_{\varphi(0)}^{\bar{F}}$.

In what follows, we suppose that the multimap $F:[\sigma, T+\sigma] \times \mathcal{B} \rightarrow K v(E)$ satisfies conditions $\left(\mathrm{F}_{1}\right),\left(\mathrm{F}_{2}\right),\left(\mathrm{F}_{4}\right)$ and $\left(\mathrm{F}_{3}^{\prime}\right)$ instead of $\left(\mathrm{F}_{3}\right)$.

Lemma 3.10. There exists a nonempty compact convex subset $X \subset D(T, \varphi)$ such that $X \supset \Sigma_{\varphi(0)}^{F}$ and, for every $t \in[\sigma, \sigma+T]$,

$$
\begin{aligned}
\left\{S(f)(t): f \in L^{1}([\sigma, \sigma+T] ; E) \text { and } f(t) \in \overline{\operatorname{co}} F\left(t, X[\varphi]_{t}\right)\right. & \\
& \text { for a.e. } t \in[\sigma, \sigma+T]\} \subset X(t),
\end{aligned}
$$

where $X(t)=\{x(t): x \in X\}$.

Proof. Set $X^{0}=\{x \in D(\varphi, T):\|x\| \leq \xi\}$, where $\xi=\max _{t \in[\sigma, T+\sigma]}\|S(0)(t)\|+$ $M\|\bar{\alpha}(\cdot)\|_{L^{1}\left([\sigma, \sigma+T] ; \mathbb{R}^{+}\right)}, M$ from condition $\left(\mathrm{S}_{1}\right)$, and $\bar{\alpha}(\cdot)$ from $\left(\mathrm{F}_{3}^{\prime}\right)$. It is clear that $X^{0}$ is a closed convex subset of $D(\varphi, T)$, and by means of $\left(1 \mathrm{~S}_{1}\right)$ (see Lemma 3.7), we have $X^{0} \supset \Sigma_{\varphi(0)}^{F}$.

Define $X^{n}=\overline{\operatorname{co}} Y^{n}$, for $n \geq 1$, where $Y^{n}$ is given by

$$
\begin{aligned}
& Y^{n}=\left\{y \in D(\varphi, T): y=S(f) ; f \in L^{1}([\sigma, T+\sigma] ; E) ;\right. \\
& \left.\quad f(t) \in \overline{\mathrm{co}} F\left(t, X^{n-1}[\varphi]_{t}\right) \text { for a.e. } t \in[\sigma, \sigma+T]\right\}, \quad n=1,2, \ldots .
\end{aligned}
$$

As $\Sigma_{\varphi(0)}^{F} \subset X^{n}, n \geq 0$, the sets $X^{n}, n \geq 0$, are nonempty convex closed subsets of $D(\varphi, T)$. Moreover, by construction, we have that $X^{n} \subset X^{n-1}, n \geq 1$.

Let us define on bounded subsets of $D(\varphi, T)$ a measure of noncompactness $\Psi$ as follows,

$$
\Psi(\Omega)=\max _{\mathcal{D} \in \Delta(\Omega)} \sup _{t \in[\sigma, T+\sigma]} e^{-L(t-\sigma)} \chi(\mathcal{D}(t)),
$$

where $\Delta(\Omega)$ is the collection of all denumerable subsets of $\Omega, \mathcal{D}(t)=\{x(t)$ : $x \in \mathcal{D}\}$, and $L>0$ a constant, chosen so that

$$
q=\sup _{t \in[\sigma, T+\sigma]} 4 M \int_{\sigma}^{t} e^{-L(t-s)} \kappa(s) d s \leq \frac{1}{2},
$$


where $\kappa(\cdot)$ is from condition $\left(\mathrm{F}_{4}\right)$. The range for the function $\Psi$ is $\mathbb{R}_{+}$. It is known (see [25, Example 2.1.4 ]), that $\Psi$ is well-defined monotone and nonsingular measure of noncompactness.

For $t \in[\sigma, T+\sigma]$, using expression (3.1), we immediately have that, for every bounded set $\Omega \subset D(\varphi, T)$,

$$
\begin{array}{r}
\sup _{-\infty \leq \theta \leq 0} \chi\left(\Omega[\varphi]_{t}(\theta)\right) \leq \sup _{-\infty<\theta \leq \sigma-t} \chi(\varphi(t-\sigma+\theta))+\sup _{\sigma-t \leq \theta \leq 0} \chi(\Omega(t+\theta)) \\
=\sup _{\sigma \leq \theta \leq t} \chi(\Omega(\theta)) .
\end{array}
$$

Hence, by $\left(\mathrm{F}_{4}\right)$, for every bounded set $\Omega \subset D(\varphi, T)$,

$$
\chi\left(F\left(t, \Omega[\varphi]_{t}\right)\right) \leq \kappa(t) \sup _{\sigma \leq \theta \leq t} \chi(\Omega(\theta)), \quad \text { for a.e. } t \in[\sigma, T+\sigma] .
$$

We claim that $\Psi\left(X^{n}\right) \stackrel{n \rightarrow \infty}{\longrightarrow} 0$. Remark first that $\Psi\left(X^{n}\right)=\Psi\left(\overline{\mathrm{co}} Y^{n}\right)=\Psi\left(Y^{n}\right)$, for $n \geq 1$. Let the maximum on the left-hand side of inequality (3.12), with $Y^{n}$ instead of $\Omega$, be achieved for a countable set $D^{\prime}=\left\{z^{m}\right\}_{m=1}^{\infty} \subset Y^{n}$. Then, $z^{m}=S\left(f_{m}\right), m \geq 1$, where $\left\{f_{m}\right\}_{m} \subset L^{1}([\sigma, T+\sigma] ; E)$ is a sequence such that, for almost every $t \in[\sigma, T+\sigma], f_{m}(t) \in \overline{\mathrm{co}} F\left(t, X^{n-1}[\varphi]_{t}\right)$, for $m \geq 1$.

For simplicity, if $\widetilde{\Omega}$ is a subset of $E$, we denote also by $\Delta(\widetilde{\Omega})$ the collection of all denumerable subsets of $\widetilde{\Omega}$. Using (3.14) and Lemma 2.2, we have, for almost every $t \in[\sigma, T+\sigma]$,

$$
\begin{aligned}
\chi\left(\left\{f_{m}(t)\right\}_{m=1}^{\infty}\right) & \leq \chi\left(\overline{\operatorname{co}} F\left(t, X^{n-1}[\varphi]_{t}\right)=\chi\left(F\left(t, X^{n-1}[\varphi]_{t}\right)\right)\right. \\
& \leq \kappa(t) \sup _{\sigma \leq \theta \leq t} \chi\left(X^{n-1}(\theta)\right) \leq \kappa(t) \sup _{\sigma \leq \theta \leq t} 2 \sup _{\widetilde{\mathcal{D}} \in \Delta\left(X^{n-1}(\theta)\right)} \chi(\widetilde{\mathcal{D}}) \\
& =2 \kappa(t) \sup _{\sigma \leq \theta \leq t \mathcal{D} \in \Delta\left(X^{n-1}\right)} \chi(\mathcal{D}(\theta)) \\
& \leq 2 \kappa(t) \sup _{\sigma \leq \theta \leq t}\left[\sup _{\mathcal{D} \in \Delta\left(X^{n-1}\right) \sigma \leq \theta \leq t} \sup _{\sigma \leq t} \chi(\mathcal{D}(\theta))\right] \\
& \left.=2 \kappa(t) \sup _{\mathcal{D} \in \Delta\left(X^{n-1}\right) \sigma \leq \theta \leq t} \sup _{\sigma \leq \theta} \mathcal{D}(\theta)\right) \\
& \leq 2 e^{L(t-\sigma)} \kappa(t) \sup _{\mathcal{D} \in \Delta\left(X^{n-1}\right) \sigma \leq \theta \leq t} \sup ^{-L(\theta-\sigma)} \chi(\mathcal{D}(\theta)) \\
& =2 e^{L(t-\sigma)} \kappa(t) \Psi\left(X^{n-1}\right) .
\end{aligned}
$$

Due to Lemma 3.8 (a) and (3.13), we have, for all $t \in[\sigma, T+\sigma]$,

$$
\begin{aligned}
e^{-L(t-\sigma)} \chi\left(\left\{z^{m}(t)\right\}_{m=1}^{\infty}\right) & =e^{-L(t-\sigma)} \chi\left(S\left\{f_{m}(t)\right\}_{m=1}^{\infty}\right) \\
& \leq 4 M e^{-L(t-\sigma)} \int_{\sigma}^{t} e^{L(s-\sigma)} k(s) d s \Psi\left(X^{n-1}\right) \\
& \leq q \Psi\left(X^{n-1}\right) \leq \frac{1}{2} \Psi\left(X^{n-1}\right) .
\end{aligned}
$$


Hence, $\Psi\left(X^{n}\right)=\Psi\left(Y^{n}\right) \leq \Psi\left(X^{n-1}\right) / 2$, for $n \geq 1$. Therefore

$$
\Psi\left(X^{n}\right) \rightarrow 0 \quad \text { when } n \rightarrow \infty .
$$

This proves that our claim is true.

Now let us consider the set $\widetilde{X}=\bigcap_{n \geq 0} X^{n} \subset D(\varphi, T)$. Because $\Sigma_{\varphi(0)}^{F} \subset \widetilde{X}$, the set $\widetilde{X}$ is nonempty closed and convex. Moreover, from the monotonicity of $\Psi$, we have $\Psi(\widetilde{X})=0$, hence $\chi(\widetilde{X}(t))=0$, for all $t \in[\sigma, T+\sigma]$. Thus by (3.14), we obtain

$$
\chi\left(\overline{\mathrm{co}} F\left(t, \tilde{X}[\varphi]_{t}\right)\right)=\chi\left(F\left(t, \widetilde{X}[\varphi]_{t}\right)\right)=0, \quad \text { for all } t \in[\sigma, T+\sigma] .
$$

The set $X \subset \tilde{X}, X=\overline{\operatorname{co}} \tilde{Y}$, where

$$
\begin{aligned}
& \widetilde{Y}=\left\{y \in D(\varphi, T): y=S(f) ; f \in L^{1}([\sigma, T+\sigma] ; E)\right. \\
& \left.\qquad f(t) \in \overline{\operatorname{co}} F\left(t, \widetilde{X}[\varphi]_{t}\right) \text { for a.e. } t \in[\sigma, T+\sigma]\right\},
\end{aligned}
$$

is compact. Indeed, consider any arbitrary sequence $\left\{y_{m}\right\}_{m=1}^{\infty} \subset \widetilde{Y}$ with $y_{m}=$ $S\left(f_{m}\right), f_{m}(t) \in \overline{\operatorname{co}} F\left(t, \tilde{X}[\varphi]_{t}\right)$, for almost every $t \in[\sigma, T+\sigma]$. From (3.16), we have, $\chi\left(\left\{f_{m}(t)\right\}_{m=1}^{\infty}\right)=0$, for almost every $t \in[\sigma, T+\sigma]$, and by $\left(\mathrm{F}_{3}^{\prime}\right)$, the sequence $\left\{f_{m}\right\}_{m=1}^{\infty}$ is integrably bounded. Then $\left\{f_{m}\right\}_{m=1}^{\infty}$ is semicompact in $L^{1}([\sigma, T+\sigma] ; E)$, by Lemma 2.3, we have $f_{m} \underset{m \rightarrow \infty}{\stackrel{\mathrm{w}}{\longrightarrow}} f_{0}$. Applying Lemma $3.8(\mathrm{~b})$, we get that

$$
y_{m}=S\left(f_{m}\right) \stackrel{m \rightarrow \infty}{\longrightarrow} y_{0}=S\left(f_{0}\right) .
$$

Let us show that $f_{0}(t) \in \overline{\mathrm{co}} F\left(t, \tilde{X}[\varphi]_{t}\right)$, for a.e. $t \in[\sigma, T+\sigma]$. Thanks to Mazur's lemma (see, for example [6]) there exists a double sequence of nonnegative numbers $\left\{\alpha_{i k}\right\}_{i=1}^{\infty}$ such that $\Sigma_{k=i}^{\infty} \alpha_{i k}=1$ for all $i=1,2, \ldots$; for every $i=1,2, \ldots$. there exists a number $k_{0}(i)$ such $\alpha_{i k}=0$ for all $k \geq k_{0}(i)$; the sequence $\left\{\widetilde{f}_{i}\right\}_{i=1}^{\infty}$;

$$
\tilde{f}_{i}(t)=\sum_{k=i}^{\infty} \alpha_{i k} f_{k}(t)
$$

converges to $f_{0}$ with the respect to the norm of the space $L^{1}([\sigma, T+\sigma] ; E)$ compact. We can extract a subsequence of $\left\{\widetilde{f}_{i}\right\}_{i=1}^{\infty}$ which converges to $f_{0}$ almost everywhere on $[\sigma, T+\sigma]$. For simplicity, we denote this extracted sequence by $\left\{\tilde{f}_{i}\right\}_{i=1}^{\infty}$. We have

$$
f_{i}(t) \in \overline{\operatorname{co}} F\left(t, \tilde{X}[\varphi]_{t}\right), \quad \text { for a.e. } t \in[\sigma, T+\sigma] \text { and for all } i \geq 1 \text {. }
$$

Then

$$
\widetilde{f}_{i}(t) \in \overline{\operatorname{co}} F\left(t, \tilde{X}[\varphi]_{t}\right), \quad \text { for a.e. } t \in[\sigma, T+\sigma] \text { and for all } i \geq 1 \text {. }
$$

Consequently, $f_{0}(t) \in \overline{\operatorname{co}} F\left(t, \tilde{X}[\varphi]_{t}\right)$, for almost every $t \in[\sigma, T+\sigma]$. Hence $\tilde{Y}$ is relatively compact. Therefore $X=\overline{\mathrm{co}} \widetilde{Y}$ is compact. By construction, the set $X$ is the required one. 
Corollary 3.11. The set $\Sigma_{\varphi(0)}^{F}$ is compact.

Proof. Indeed, taking into account Lemma 3.10, it is enough to show its closeness. Let $\left\{x_{m}\right\}_{m=1}^{\infty} \subset \Sigma_{\varphi(0)}^{F}$ be an arbitrary sequence with $x_{m}=S\left(f_{m}\right)$, $f_{m}(t) \in F\left(t, x_{m}[\varphi]_{t}\right)$, for almost every $t \in[\sigma, T+\sigma]$ and $x_{m} \stackrel{m \rightarrow \infty}{\longrightarrow} x_{0}$. Arguing as above, one can easily show that the sequence $\left\{f_{m}\right\}_{m=1}^{\infty}$ is semicompact in $L^{1}([\sigma, T+\sigma] ; E)$, by Lemma 2.3 , we have that $f_{m} \underset{m \rightarrow \infty}{\stackrel{\mathrm{w}}{\longrightarrow}} f_{0}$. Lemma $3.8(\mathrm{~b})$ together with Lemma 3.4 imply that $x_{m}=S\left(f_{m}\right) \stackrel{m \rightarrow \infty}{\longrightarrow} x_{0}=S\left(f_{0}\right)$ with $f_{0} \in \overline{\operatorname{sel}}_{F}\left(x_{0}\right)$. Hence $x_{0} \in \Sigma_{\varphi(0)}^{F}$.

Lemma 3.12. The set $X[\varphi]_{(\cdot)}=\left\{x[\varphi]_{(\cdot)}: t \rightarrow x[\varphi]_{t} ; x \in X\right\} \subset C([\sigma, T+\sigma] ; \mathcal{B})$ is compact.

Proof. Let us consider the map $\zeta$ defined as

$$
\zeta: D(\varphi, T) \rightarrow C([\sigma, T+\sigma] ; \mathcal{B}), \quad \zeta(x)=x[\varphi]_{(\cdot)} .
$$

By axiom (B2), we have

$$
\begin{aligned}
\sup _{t \in[\sigma, T+\sigma]}\left\|x[\varphi]_{t}-y[\varphi]_{t}\right\|_{\mathcal{B}} & \leq \beta_{K} \sup _{t \in[\sigma, T+\sigma]} \sup _{s \in[\sigma, t]}\|x(s)-y(s)\| \\
& \leq \beta_{K}\|x(\cdot)-y(\cdot)\|_{C([\sigma, T+\sigma] ; E)},
\end{aligned}
$$

where $\beta_{K}$ is from (3.10). As a consequence $\zeta$ is continuous. Therefore $\zeta(X)=$ $X[\varphi]_{(\cdot)}$ is a compact set in $C([\sigma, T+\sigma] ; \mathcal{B})$.

COROLlary 3.13. From Lemma 2.1, it results immediately that the application,

$$
X[\varphi]_{(\cdot)}:[\sigma, T+\sigma] \rightarrow K(\mathcal{B}), \quad t \mapsto X[\varphi]_{t},
$$

is continuous and the set $X[\varphi]_{[\sigma, T+\sigma]} \subset \mathcal{B}$ is compact.

Now consider the metric projection $P:[\sigma, T+\sigma] \times \mathcal{B} \rightarrow K v(\mathcal{B})$,

$$
P(t, u)=\left\{v \in X[\varphi]_{t}:\|v-u\|=\operatorname{dist}\left(u, X[\varphi]_{t}\right)\right\},
$$

where $\left.\operatorname{dist}\left(u, X[\varphi]_{t}\right)\right\}=\inf \left\{\|u-\widetilde{v}\|, \widetilde{v} \in X[\varphi]_{t}\right\}$. Let $\widehat{F}:[\sigma, T+\sigma] \times \mathcal{B} \rightarrow K v(E)$ be defined by

$$
\widehat{F}(t, u)=\overline{\mathrm{co}} F(t, P(t, u)) .
$$

Bearing in mind the results of the previous corollary and arguing exactly by the same reasoning as in the proof of [25, Lemma 5.3.2], one can easily show that the multimap $\widehat{F}$ satisfies $\left(\mathrm{F}_{1}\right),\left(\mathrm{F}_{2}\right)$ and $\left(\mathrm{F}_{4}\right)$, condition $\left(\mathrm{F}_{3}^{\prime}\right)$ is obviously satisfied by $\widehat{F}$. Hence taking $\widehat{F}$ instead of $F$ in Lemma 3.5 and Corollary 3.11, we deduce that under the conditions of Theorem 3.1, the solution set $\Sigma_{\varphi(0)}^{\widehat{F}}$ of the problem

is a compact set.

$$
x \in S \circ \overline{\operatorname{sel}}_{\widehat{F}}(x)
$$


LEMmA 3.14. $\Sigma_{\varphi(0)}^{F}=\Sigma_{\varphi(0)}^{\widehat{F}}$.

Proof. Let $x \in \Sigma_{\varphi(0)}^{\widehat{F}}$. Then

$$
\begin{aligned}
x(t) \in & \left\{S(g)(t): g \in L^{1}([\sigma, T+\sigma] ; E), g(t) \in \widehat{F}\left(t, x[\varphi]_{t}\right) \text { a.e. } t \in[\sigma, T+\sigma]\right\} \\
= & \left\{S(g)(t): g \in L^{1}([\sigma, T+\sigma], E), g(t) \in \overline{\operatorname{co}} F\left(t, P\left(t, x[\varphi]_{t}\right)\right)\right. \\
& \text { for a.e. } t \in[\sigma, T+\sigma]\} \\
\subseteq & \left\{S(g)(t): g \in L^{1}([\sigma, T+\sigma], E), g(t) \in \overline{\operatorname{co}} F\left(t, X[\varphi]_{t}\right)\right. \\
& \quad \text { for a.e. } t \in[\sigma, T+\sigma]\} \subseteq X(t) .
\end{aligned}
$$

Consequently, $P(t, x(t))=\{x(t)\}$. Thus $x(t)=S(f)(t), t \in[\sigma, T+\sigma]$, with

$$
f(t) \in \widehat{F}\left(t, x[\varphi]_{t}\right)=\overline{\mathrm{co}} F\left(t, P\left(t, x[\varphi]_{t}\right)\right)=\overline{\mathrm{co}} F\left(t, x[\varphi]_{t}\right)=F\left(t, x[\varphi]_{t}\right),
$$

for almost every $t \in[\sigma, T+\sigma]$. Thus, $x \in \Sigma_{\varphi(0)}^{F}$. Let us prove that $\Sigma_{\varphi(0)}^{F} \subseteq \Sigma_{\varphi(0)}^{\widehat{F}}$. Let $x \in \Sigma_{\varphi(0)}^{F}$. As $\Sigma_{\varphi(0)}^{F} \subset X$, we have, for every $t \in[\sigma, T+\sigma], x[\varphi]_{t} \in X[\varphi]_{t}$. Hence, for every $t \in[\sigma, T+\sigma], P\left(t, x[\varphi]_{t}\right)=\left\{x[\varphi]_{t}\right\}$. As a consequence,

$$
x=S(g), \quad g(t) \in F\left(t, x[\varphi]_{t}\right)=F\left(t, P\left(t, x[\varphi]_{t}\right)\right)=\widehat{F}\left(t, x[\varphi]_{t}\right),
$$

for almost every $t \in[\sigma, T+\sigma]$. Thus, $x \in \Sigma_{\varphi(0)}^{\widehat{F}}$.

Lemma 3.15. There exists a sequence of multimaps $\left\{F_{n}\right\}, F_{n}:[\sigma, T+\sigma] \times \mathcal{B} \rightarrow$ $K v(E)$ such that:

(a) each multimap $F_{n}(t, \cdot): \mathcal{B} \rightarrow K v(E)$ is continuous for almost every $t \in$ $[\sigma, \sigma+T]$

(b) $\widehat{F}(t, u) \subset \ldots \subset F_{n+1}(t, u) \subset F_{n}(t, u) \subset \overline{\operatorname{co}} F\left(t, X[\varphi]_{t}\right), n \geq 1$;

(c) $\widehat{F}(t, u)=\bigcap_{n \geq 1} F_{n}(t, u)$;

(d) for each $n \geq 1$, there exists a selection $g_{n}(t, u) \in F_{n}(t, u)$ such that $g_{n}(\cdot, u)$ is measurable and $g_{n}(t, \cdot)$ is locally Lipschitz in the sense that, for every compact $\mathfrak{K} \subset \mathcal{B}$, there exist $c_{1}(\mathfrak{K})>0$ and $c_{2}(\mathfrak{K})>0$, such that for almost every $t \in[\sigma, T+\sigma]$ and for all $u, v \in \overline{\mathfrak{K}}_{c_{2}(\mathfrak{K})}$,

$$
\left\|g_{n}(t, u)-g_{n}(t, v)\right\| \leq c_{1}(\mathcal{K}) \bar{\alpha}(t)\|u-v\|_{\mathcal{B}},
$$

where $\overline{\mathfrak{K}}_{c_{2}(\mathcal{K})}$ denotes the closure of the $c_{2}(\mathfrak{K})$-neighbourhood of $\mathfrak{K}$ and $\bar{\alpha}(\cdot)$ is from $\left(\mathrm{F}_{3}^{\prime}\right)$.

Since the space $\mathcal{B}$ is supposed to be a Banach space, the proof of Lemma 3.15 follows, except for some obvious modifications, the same lines as in the proof of [25, Lemma 5.3.4] (see also [13, Theorem 7.2], from where this approximation is taken). We do not give details.

LEMMA 3.16. For each $n \geq 1$, the set $\Sigma_{\varphi(0)}^{F_{n}}$ is compact contractible. 
The proof of this lemma is obtained from five auxiliary lemmas. Note first that from (a), (b) and (d) of Lemma 3.15, it follows that each $F_{n}, n \geq 1$, satisfies conditions $\left(\mathrm{F}_{1}\right),\left(\mathrm{F}_{2}\right),\left(\mathrm{F}_{3}^{\prime}\right)$ and $\left(\mathrm{F}_{4}\right)$. Hence, according to Lemma 3.5 and Corollary 3.11, under conditions of Theorem 3.1, for each $n \geq 1$, the solution set $\Sigma_{\varphi(0)}^{F_{n}}$ of the problem

$$
x \in S \circ \overline{\operatorname{sel}}_{F_{n}}(x)
$$

is a compact subset of $D(\varphi, T)$, where $\overline{\operatorname{sel}}_{F_{n}}(\cdot)$ is given by $(3.3)$ with $F_{n}$ instead of $F$.

Fix $n \geq 1$. Let $x_{n} \in \Sigma_{\varphi(0)}^{F_{n}}$, with $x_{n}=S\left(f_{n}\right)$ for some $f_{n} \in \overline{\operatorname{sel}}_{F_{n}}\left(x_{n}\right)$. For each $\tau \in[0,1)$, let $D^{\tau}\left(x_{n}, T\right)$ be a set defined by

$$
D^{\tau}\left(x_{n}, T\right)=\left\{x \in D(\varphi, T): x(t)=x_{n}(t), \text { for all } t \in[\sigma, \tau T+\sigma]\right\} .
$$

It is clear that for each $\tau \in[0,1)$, the set $D^{\tau}\left(x_{n}, T\right)$ is a closed (convex ) subset of $D(\varphi, T)$. Let $\widehat{f}:[\sigma, T+\sigma] \times \mathcal{B} \rightarrow E$ be defined by

$$
\widehat{f}(t, u)=\mathbf{1}_{[\sigma, \tau T+\sigma]} f_{n}(t)+\mathbf{1}_{[\tau T+\sigma, T+\sigma]} g_{n}(t, u),
$$

where $g_{n}$ is from Lemma $3.15(\mathrm{~d})$. It is clear that for any $x \in D^{\tau}\left(x_{n}, T\right)$, we have

$$
\overline{\operatorname{sel}}_{\widehat{f}}(x)=\mathbf{1}_{[\sigma, \tau T+\sigma]} f_{n}(\cdot)+\mathbf{1}_{[\sigma+\tau T, T+\sigma]}(\cdot) g_{n}(\cdot, x[\varphi] .) .
$$

Lemma 3.17. For every $x \in D^{\tau}\left(x_{n}, T\right)$, we have

$$
\mathbf{1}_{[\sigma, \tau T+\sigma]} f_{n}(t)+\mathbf{1}_{[\tau T+\sigma, T+\sigma]} g_{n}\left(t, x[\varphi]_{t}\right) \in F_{n}\left(t, x[\varphi]_{t}\right),
$$

for almost every $t \in[\sigma, T+\sigma]$.

Proof. Since $x \in D^{\tau}\left(x_{n}, T\right)$, we have $x(t)=x_{n}(t)$, for all $t \in[\sigma, \tau T+\sigma]$. Thus

$$
f_{n}(t) \in F_{n}\left(t, x_{n}[\varphi]_{t}\right)=F_{n}\left(t, x[\varphi]_{t}\right), \quad \text { for a.e. } t \in[\sigma, \tau T+\sigma] .
$$

Now, from Lemma 3.15 (d), we have

$$
g_{n}\left(t, x[\varphi]_{t}\right) \in F_{n}\left(t, x[\varphi]_{t}\right), \quad \text { for a.e. } t \in[\tau T+\sigma, T+\sigma] .
$$

Expression (3.21) together with (3.22) imply (3.20).

Let $\mathcal{F}_{n}^{\tau}: D^{\tau}\left(x_{n}, T\right) \rightarrow D^{\tau}\left(x_{n}, T\right)$ be defined by

$$
\mathcal{F}_{n}^{\tau}(x)=S \circ \overline{\operatorname{sel}}_{\widehat{f}}(x) .
$$

Observe that, for every $x \in D^{\tau}\left(x_{n}, T\right)$, one can write,

$$
\overline{\operatorname{sel}}_{\widehat{f}}(x)=\mathbf{1}_{[\sigma, \tau T+\sigma]} f_{n}+\mathbf{1}_{[\sigma+\tau T, T+\sigma]} G_{n}^{\tau}(x),
$$

with

$$
G_{n}^{\tau}(x)(t)= \begin{cases}0 & \text { for } t \in[\sigma, \tau T+\sigma[ \\ g_{n}\left(t, x[\varphi]_{t}\right) & \text { for } t \in[\sigma+\tau T, T+\sigma]\end{cases}
$$


Thus, for every $x \in D^{\tau}\left(x_{n}, T\right)$, we have

$$
\mathcal{F}_{n}^{\tau}(x)=S\left(\mathbf{1}_{[\sigma, \tau T+\sigma]} f_{n}+\mathbf{1}_{[\sigma+\tau T, T+\sigma]} G_{n}^{\tau}(x)\right) .
$$

Lemma 3.18. For each $\tau \in[0,1)$, the map $\mathcal{F}_{n}^{\tau}$ is well-defined and maps $D^{\tau}\left(x_{n}, T\right)$ into itself.

ProOF. From property $\left(2 \mathrm{~S}_{1}\right)$ of $S$ (see Lemma 3.7 ), the value $\mathcal{F}_{n}^{\tau}(x)$ does not depend on the choice of $f_{n}$. Hence $\mathcal{F}_{n}^{\tau}$ is well-defined.

Using property $\left(1 \mathrm{~S}_{1}\right.$ ) (see Lemma 3.7), we obtain, for all $x \in D^{\tau}\left(x_{n}, T\right)$,

$$
\begin{aligned}
\left\|\mathcal{F}_{n}^{\tau}(x)(t)-x_{n}(t)\right\| & =\left\|\mathcal{F}_{n}^{\tau}(x)(t)-S\left(f_{n}\right)(t)\right\| \\
& =\left\|S\left(\mathbf{1}_{[\sigma, \tau T+\sigma]} f_{n}+\mathbf{1}_{[\sigma+\tau T, T+\sigma]} G_{n}^{\tau}(x)\right)(t)-S\left(f_{n}\right)(t)\right\| \\
& =M \int_{\sigma}^{t}\left\|\mathrm{f}(\gamma)-\mathbf{1}_{[\sigma, \tau T+\sigma]} \mathrm{f}(\gamma)\right\| d \gamma=0,
\end{aligned}
$$

for all $t \in[\sigma, \tau T+\sigma]$. Hence, for all $x \in D^{\tau}\left(x_{n}, T\right)$, we have

$$
\mathcal{F}_{n}^{\tau}(x)(t)=x_{n}(t), \quad \text { for all } t \in[\sigma, \tau T+\sigma] .
$$

Consequently, $\mathcal{F}_{n}^{\tau}$ maps $D^{\tau}\left(x_{n}, T\right)$ onto itself.

Now, for each $\tau \in[0,1)$, let us consider in $D^{\tau}\left(x_{n}, T\right)$ the problem

$$
x=\mathcal{F}_{n}^{\tau}(x)=S \circ \overline{\operatorname{sel}}_{\widehat{f}}(x) .
$$

LEMMA 3.19. Every local solution of problem (3.24) is a local solution of problem (3.19) (see Definition 1.2).

Proof. Let $z \in D^{\tau}\left(x_{n}, T\right)$, such that $\left.z\right|_{[\sigma, \sigma+b]}$ is a local solution of (3.24) for some $0<b \leq T$. Then

$$
\left.z(\cdot)\right|_{[\sigma, \sigma+b]}=\left.S\left(\mathbf{1}_{[\sigma, \tau T+\sigma]} f_{n}+\mathbf{1}_{[\sigma+\tau T, T]} G_{n}^{\tau}(z)\right)(\cdot)\right|_{[\sigma, \sigma+b]} \cdot
$$

Expression (3.20) together with (3.25) imply that $z$ is a local solution of (3.19).

Lemma 3.20. For each $\tau \in[0,1)$, problem (3.24) has a local solution defined on $[\sigma, \tau T+\sigma+h]$ for some $h>0$ small enough.

Proof. Let us fix $\tau \in[0,1)$. Consider the function $\bar{\varphi}(t)$ given by

$$
\bar{\varphi}(t)= \begin{cases}x_{n}(t) & \text { for } t \in[\sigma, \tau T+\sigma], \\ x_{n}(\sigma+\tau T) & \text { for } t \in[\tau T+\sigma, T+\sigma] .\end{cases}
$$

It is clear that $\bar{\varphi} \in D^{\tau}\left(x_{n}, T\right)$. The set

$$
\mathcal{Q}=\varrho([\sigma, T+\sigma], \bar{\varphi})=\left\{\bar{\varphi}[\varphi]_{t}: t \in[\sigma, T+\sigma]\right\} \subset \mathcal{B}
$$

is compact, where $\varrho$ is from $(3.2)$. Let $c_{1}(\mathcal{Q}), c_{2}(\mathcal{Q})>0$ be such that $(3.18)$ holds with $\mathfrak{K}=\mathcal{Q}$, and let $r>0$ be chosen so that

$$
r \beta_{K} \leq c_{2}(\mathcal{Q})
$$


where $\beta_{K}$ is from (3.10). By $\overline{\mathcal{Q}_{r \beta_{K}}}$ denote the closure of the $r \beta_{K}$-neighbourhood of $\mathcal{Q}$. Let $h>0$ be chosen so that

$$
\begin{aligned}
& \qquad \int_{\sigma+\tau T}^{\sigma+\tau T+h} \bar{\alpha}(s) d s \leq \frac{r}{4 M}, \quad h \leq T(1-\tau) \quad \text { and } \\
& \max _{\left|t_{1}-t_{2}\right| \leq h}\left\|x_{n}\left(t_{1}\right)-x_{n}\left(t_{2}\right)\right\| \leq r / 2, \quad t_{1}, t_{2} \in[\sigma, T+\sigma], \\
& \text { from the estimate }\left(\mathrm{F}_{3}^{\prime}\right) \text { and } M \text { from condition }\left(\mathrm{S}_{1}\right) .
\end{aligned}
$$

where $\bar{\alpha}$ is from the estimate $\left(\mathrm{F}_{3}^{\prime}\right)$ and $M$ from condition $\left(\mathrm{S}_{1}\right)$.

Consider the map $\widehat{\mathcal{F}_{n}^{\tau}}:\left.\left.D^{\tau}\left(x_{n}, T\right)\right|_{[\sigma, \tau T+\sigma+h]} \rightarrow D^{\tau}\left(x_{n}, T\right)\right|_{[\sigma, \tau T+\sigma+h]}$, defined by

$$
\begin{aligned}
\widehat{\mathcal{F}}_{n}^{\tau}\left(\left.x\right|_{[\sigma, \tau T+\sigma+h]}\right) & =\left.\mathcal{F}_{n}^{\tau}(x)\right|_{[\sigma, \tau T+\sigma+h]} \\
& =\left.S\left(\mathbf{1}_{[\sigma, \tau T+\sigma]} f_{n}+\mathbf{1}_{[\sigma+\tau T, T+\sigma]} G_{n}^{\tau}(x)\right)\right|_{[\sigma, \tau T+\sigma+h]} ;
\end{aligned}
$$

where $\left.D^{\tau}\left(x_{n}, T\right)\right|_{[\sigma, \tau T+\sigma+h]}=\left\{\left.x\right|_{[\sigma, \tau T+\sigma+h]}: x \in D^{\tau}\left(x_{n}, T\right)\right\}$. Here, for $x \in$ $D\left(x_{n}, T\right)$, the element $\left.x\right|_{[\sigma, \tau T+\sigma+h]} \in C([\sigma, \tau T+\sigma+h] ; E)$ is the restriction of $x$ to the interval $[\sigma, \tau T+\sigma+h]$.

Before continuing the proof, let us make some remarks:

REMARK 3.21. It is clear that,

(a) the set $\left.D\left(x_{n}, T\right)\right|_{[\sigma, \tau T+\sigma+h]}$ is closed (convex) subset of $C([\sigma, \tau T+\sigma+$ $h] ; E)$;

(b) from (3.23), we have for all $x \in D^{\tau}\left(x_{n}, T\right)$ and $t \in[\sigma, \tau T+\sigma]$,

$$
\widehat{\mathcal{F}_{n}^{\tau}}\left(\left.x\right|_{[\sigma, \tau T+\sigma+h]}\right)(t)=\left.\mathcal{F}_{n}^{\tau}(x)\right|_{[\sigma, \tau T+\sigma+h]}(t)=\mathcal{F}_{n}^{\tau}(x)(t)=x_{n}(t) ;
$$

(c) for all $x \in D^{\tau}\left(x_{n}, T\right)$,

$$
\left.x\right|_{[\sigma, \tau T+\sigma+h]}[\varphi]_{t}=x[\varphi]_{t}, \quad \text { for all } t \in[\sigma, \tau T+\sigma+h] .
$$

Now, in $\left.D^{\tau}\left(x_{n}, T\right)\right|_{[\sigma, \tau T+\sigma+h]}$, denote by $\overline{B^{\tau}}\left(\left.\bar{\varphi}\right|_{[\sigma, \tau T+\sigma+h]}, r\right)$ the closed ball of the radius $r$ centered at $\left.\bar{\varphi}\right|_{[\sigma, \tau T+\sigma+h]}$, where $\bar{\varphi}$ is from (3.26), i.e.

$$
\begin{aligned}
& \overline{B^{\tau}}\left(\left.\bar{\varphi}\right|_{[\sigma, \tau T+\sigma+h]}, r\right) \\
& =\left\{\left.\left.x\right|_{[\sigma, \tau T+\sigma+h]} \in D^{\tau}\left(x_{n}, T\right)\right|_{[\sigma, \tau T+\sigma+h]}:\right. \\
& \left.\left\|\left.x\right|_{[\sigma, \tau T+\sigma+h]}-\left.\bar{\varphi}\right|_{[\sigma, \tau T+\sigma+h]}\right\|_{C([\sigma, \tau T+\sigma+h] ; E)} \leq r\right\} \\
& =\left\{\left.\left.x\right|_{[\sigma, \tau T+\sigma+h]} \in D^{\tau}\left(x_{n}, T\right)\right|_{[\sigma, \tau T+\sigma+h]}: \sup _{t \in[\sigma, \sigma+\tau T+h]}\|x(t)-\bar{\varphi}(t)\| \leq r\right\} \\
& =\left\{\left.\left.x\right|_{[\sigma, \tau T+\sigma+h]} \in D^{\tau}\left(x_{n}, T\right)\right|_{[\sigma, \tau T+\sigma+h]}:\right. \\
& \left.\sup _{t \in[\sigma+\tau T, \sigma+\tau T+h]}\left\|x(t)-x_{n}(\sigma+\tau T)\right\| \leq r\right\} .
\end{aligned}
$$

Let us prove that $\widehat{\mathcal{F}}_{n}^{\tau}$ maps $\overline{B^{\tau}}\left(\left.\bar{\varphi}\right|_{[\sigma, \tau T+\sigma+h]}, r\right)$ into itself. Take $\left.x\right|_{[\sigma, \tau T+\sigma+h]}$ in $\overline{B^{\tau}}\left(\left.\bar{\varphi}\right|_{[\sigma, \tau T+\sigma+h]}, r\right)$. Let us estimate the value

$$
\left\|\mathcal{F}_{n}^{\tau}\left(\left.x\right|_{[\sigma, \tau T+\sigma+h]}\right)-\left.\bar{\varphi}\right|_{[\sigma, \tau T+\sigma+h]}\right\| .
$$


From Remark 3.21 (b) and (3.23), we have for any $t \in[\sigma, \tau T+\sigma]$,

$$
\left\|\widehat{\mathcal{F}}_{n}^{\tau}\left(\left.x\right|_{[\sigma, \tau T+\sigma+h]}\right)(t)-\left.\bar{\varphi}\right|_{[\sigma, \tau T+\sigma+h]}(t)\right\|=\left\|x_{n}(t)-x_{n}(t)\right\|=0 .
$$

By property $\left(1 \mathrm{~S}_{1}\right)$ (see Lemma 3.7), (3.29), (3.30), (3.32), and the fact that $S\left(f_{n}\right)(\cdot)=x_{n}(\cdot)$, we obtain for any $t \in[\tau T+\sigma, \tau T+\sigma+h]$,

$$
\begin{aligned}
& \left\|\widehat{\mathcal{F}}_{n}^{\tau}\left(\left.x\right|_{[\sigma, \tau T+\sigma+h]}\right)(t)-\left.\bar{\varphi}\right|_{[\sigma, \tau T+\sigma+h]}(t)\right\| \\
& =\left\|S\left(\mathbf{1}_{[\sigma, \tau T+\sigma]} f_{n}+\mathbf{1}_{[\sigma+\tau T, T+\sigma]} G_{n}^{\tau}(x)\right)(t)-x_{n}(\sigma+\tau T)\right\| \\
& \leq\left\|S\left(\mathbf{1}_{[\sigma, \tau T+\sigma+h]} f_{n}+\mathbf{1}_{[\sigma+\tau T, T+\sigma+h]} G_{n}^{\tau}(x)\right)(t)-S\left(f_{n}\right)(t)\right\| \\
& \quad+\left\|S\left(f_{n}\right)(t)-x_{n}(\sigma+\tau T)\right\| \\
& \leq M \int_{\sigma}^{\tau T+\sigma}\left\|f_{n}(\gamma)-\mathbf{1}_{[\sigma, \tau T+\sigma]} f_{n}(\gamma)\right\| d \gamma \\
& \quad+M \int_{\sigma+\tau T}^{\sigma+\tau T+h}\left\|f_{n}(t)-g_{n}\left(\gamma, x[\varphi]_{\gamma}\right)\right\| d \gamma+\left\|x_{n}(t)-x_{n}(\sigma+\tau T)\right\| \\
& \leq 2 M \int_{\sigma+\tau T}^{\sigma+\tau T+h} \bar{\alpha}(\gamma) d \gamma+\frac{r}{2} \leq 2 M \frac{r}{4 M}+\frac{r}{2}=r .
\end{aligned}
$$

Now in the space $C([\sigma, \tau T+\sigma+h] ; E)$, let us consider the equivalent norm $\|\cdot\|_{*}$ given by

$$
\|y\|_{*}=\max _{t \in[\sigma, \tau T+\sigma+h]} e^{-\eta(t-\sigma)}\|y(t)\|
$$

where $\eta>0$ is a constant which will be determined later.

Let us prove that

$$
\widehat{\mathcal{F}}_{n}^{\tau}:\left(\overline{B^{\tau}}\left(\left.\bar{\varphi}\right|_{[\sigma, \tau T+\sigma+h]}, r\right), d_{*}\right) \rightarrow\left(\overline{B^{\tau}}\left(\left.\bar{\varphi}\right|_{[\sigma, \tau T+\sigma+h]}, r\right), d_{*}\right)
$$

is a contraction, where $d_{*}$ is the metric induced by $\|\cdot\|_{*}$.

Remark first that for $\left.z\right|_{[\sigma, \tau T+\sigma+h]} \in \bar{B}\left(\left.\bar{\varphi}\right|_{[\sigma, \tau T+\sigma+h]}, r\right)$, by axiom (B2) and expression (3.33), we obtain, for any $t \in[\sigma, \sigma+\tau T+h]$,

$$
\begin{aligned}
\left\|\left.z\right|_{[\sigma, \tau T+\sigma+h]}[\varphi]_{t}-\left.\bar{\varphi}\right|_{[\sigma, \tau T+\sigma+h]}[\varphi]_{t}\right\|_{\mathcal{B}}=\left\|z[\varphi]_{t}-\bar{\varphi}[\varphi]_{t}\right\|_{\mathcal{B}} \\
\quad \leq \beta_{K} \sup _{t \in[\sigma, \sigma+\tau T+h]}\|z(t)-\bar{\varphi}(t)\|+M(t-\sigma)\|\varphi-\varphi\|_{\mathcal{B}} \\
\leq \beta_{K} \sup _{t \in[\sigma+\tau T, \sigma+\tau T+h]}\|z(t)-\bar{\varphi}(t)\| \leq \beta_{K} r .
\end{aligned}
$$

But, for all $t \in[\sigma+\tau T, \sigma+\tau T+h], \bar{\varphi}[\varphi]_{t} \in \mathcal{Q}$, where $\mathcal{Q}$ is from (3.27). Therefore, using (3.28), we obtain, for all $t \in[\sigma, \tau T+\sigma+h]$,

$$
\left.z\right|_{[\sigma, \tau T+\sigma+h]}[\varphi]_{t}=z[\varphi]_{t} \in \bar{Q}_{\beta_{K} r} \subset \bar{Q}_{c_{2}(\mathcal{Q})} .
$$

Let $\left.x\right|_{[\sigma, \tau T+\sigma+h]},\left.y\right|_{[\sigma, \tau T+\sigma+h]} \in \overline{B^{\tau}}\left(\varphi^{*}, r\right)$. Using (3.34), we have, for any $t \in$ $[\sigma, \tau T+\sigma+h]$

$$
\begin{aligned}
& \left.x\right|_{[\sigma, \tau T+\sigma+h]}[\varphi]_{t}=x[\varphi]_{t} \in \bar{Q}_{\beta_{K} r} \subset \bar{Q}_{c_{2}(\mathcal{Q})}, \\
& \left.y\right|_{[\sigma, \tau T+\sigma+h]}[\varphi]_{t}=y[\varphi]_{t} \in \bar{Q}_{\beta_{K} r} \subset \bar{Q}_{c_{2}(\mathcal{Q})} .
\end{aligned}
$$


Hence, because $g(t, \cdot)$ is locally Lipschitz (see Lemma 3.15(d)), we have, for almost every $t \in[\sigma, \tau T+\sigma+h]$,

$$
\left\|g_{n}\left(t, x[\varphi]_{t}\right)-g_{n}\left(t, y[\varphi]_{t}\right)\right\| \leq c_{1}(\mathcal{Q}) \bar{\alpha}(t)\left\|x[\varphi]_{t}-y[\varphi]_{t}\right\|_{\mathcal{B}} .
$$

Now, for any $t \in[\sigma, \tau T+\sigma+h]$, let us estimate

$$
\left\|\widehat{\mathcal{F}}_{n}^{\tau}\left(\left.x\right|_{[\sigma, \tau T+\sigma+h]}\right)(t)-\widehat{\mathcal{F}}_{n}^{\tau}\left(\left.y\right|_{[\sigma, \tau T+\sigma+h]}\right)(t)\right\| .
$$

If $t \in[\sigma, \tau T+\sigma]$, using (3.32), we get

$$
\left\|\widehat{\mathcal{F}}_{n}^{\tau}\left(\left.x\right|_{[\sigma, \tau T+\sigma+h]}\right)(t)-\widehat{\mathcal{F}}_{n}^{\tau}\left(\left.y\right|_{[\sigma, \tau T+\sigma+h]}\right)(t)\right\|=\left\|x_{n}(t)-x_{n}(t)\right\|=0 .
$$

If $t \in[\sigma+\tau T, \tau T+\sigma+h]$, using condition $\left(1 \mathrm{~S}_{1}\right),(3.32),(3.33),(3.35)$, and the fact that $x(t)=y(t)$ for all $t \in[\sigma, \tau T+\sigma+h]$, we obtain

$$
\begin{aligned}
\mid \widehat{\mathcal{F}}_{n} & \left(\left.x\right|_{[\sigma, \tau T+\sigma+h]}\right)(t)-\widehat{\mathcal{F}}_{n}^{\tau}\left(\left.y\right|_{[\sigma, \tau T+\sigma+h]}\right)(t)\|=\| \mathcal{F}_{n}^{\tau}(x)(t)-\mathcal{F}_{n}^{\tau}(y)(t) \| \\
\leq & \| S\left(\mathbf{1}_{[\sigma, \tau T+\sigma]} f_{n}+\mathbf{1}_{[\sigma+\tau T, T+\sigma]} G_{n}^{\tau}(x)\right)(t) \\
& -S\left(\mathbf{1}_{[\sigma, \tau T+\sigma]} f_{n}+\mathbf{1}_{[\sigma+\tau T, T+\sigma]} G_{n}^{\tau}(y)\right)(t) \| \\
\leq & M \int_{\sigma}^{\tau T+\sigma}\left\|\mathbf{1}_{[\sigma, \tau T+\sigma]} f_{n}(\gamma)-\mathbf{1}_{[\sigma, \tau T+\sigma]} f_{n}(\gamma)\right\| d \gamma \\
& +M \int_{\sigma+\tau T}^{t}\left\|g_{n}\left(\gamma, x[\varphi]_{\gamma}\right)-g_{n}\left(\gamma, y[\varphi]_{\gamma}\right)\right\| d \gamma \\
\leq & c_{1}(\mathcal{Q}) M \int_{\sigma+\tau T}^{t} \bar{\alpha}(\gamma)\left\|x[\varphi]_{\gamma}-y[\varphi]_{\gamma}\right\|_{\mathcal{B}} d \gamma \\
\leq & c_{1}(\mathcal{Q}) M \int_{\sigma+\tau T}^{t} \bar{\alpha}(\gamma)\left[\beta_{K} \sup _{\sigma \leq s \leq \gamma}\|x(s)-y(s)\|+\beta_{N}\left\|x[\varphi]_{\sigma}-y[\varphi]_{\sigma}\right\|\right] d \gamma \\
\leq & \beta_{K} c_{1}(\mathcal{Q}) M \int_{\sigma+\tau T}^{t} \bar{\alpha}(\gamma) e^{\eta(\gamma-\sigma)} e^{-\eta(\gamma-\sigma)} \sup _{\sigma+\tau T \leq s \leq \gamma}\|x(s)-y(s)\| d \gamma \\
\leq & \beta_{K} c_{1}(\mathcal{Q}) M \int_{\sigma+\tau T}^{t} \bar{\alpha}(\gamma) e^{\eta(t-\sigma)} \sup _{\sigma+\tau T \leq s \leq \gamma} e^{-\eta(s-\sigma)}\|x(s)-y(s)\| d \gamma,
\end{aligned}
$$

where $\bar{\alpha}(\cdot)$ from is condition $\left(\mathrm{F}_{3}^{\prime}\right), \beta_{K}$ is from (3.10) and $\beta_{N}$ is form (3.11). Thus

$$
\begin{aligned}
\sup _{\sigma+\tau T \leq t \leq \tau T+\sigma+h} & e^{-\eta(t-\sigma)}\left\|\widehat{\mathcal{F}}_{n}^{\tau}\left(\left.x\right|_{[\sigma+\tau T, T+\sigma]}\right)(t)-\widehat{\mathcal{F}}_{n}^{\tau}\left(\left.y\right|_{[\sigma+\tau T, T+\sigma]}\right)(t)\right\| \\
\leq & \sup _{t \in[\sigma+\tau T, T+\sigma]}\left[M \beta_{K} c_{1}(\mathcal{Q}) \int_{\sigma}^{t} \bar{\alpha}(\gamma) e^{-\eta(t-\gamma)} d \gamma\right] \\
& \cdot \sup _{\sigma+\tau T \leq s \leq \tau T+\sigma+h} e^{-\eta(s-\sigma)}\|x(s)-y(s)\| d \gamma .
\end{aligned}
$$

Observe that

$$
\sup _{t \in[\sigma, T+\sigma]}\left[M \beta_{K} c_{1}(\mathcal{Q}) \int_{\sigma}^{t} \bar{\alpha}(\gamma) e^{-\eta(t-\gamma)} d \gamma\right] \longrightarrow 0,
$$


when $\eta \rightarrow+\infty$. Choose $\eta>0$, so that

$$
\widehat{q}=\sup _{t \in[\sigma, T+\sigma]}\left[M \beta_{K} c_{1}(\mathcal{Q}) \int_{\sigma}^{t} e^{-\eta(t-\gamma)} d \gamma\right]<1 .
$$

Hence, we obtain

$$
\begin{aligned}
\sup _{\sigma+\tau T \leq s \leq \tau T+\sigma+h} e^{-\eta(s-\sigma)}\left\|\left.\widehat{\mathcal{F}}_{n}^{\tau}(x)\right|_{[\sigma+\tau T, T+\sigma]}-\left.\widehat{\mathcal{F}}_{n}^{\tau}(y)\right|_{[\sigma+\tau T, T+\sigma]}\right\| \\
\quad \leq \widehat{q} \sup _{\sigma+\tau T \leq s \leq \tau T+\sigma+h} e^{-\eta(s-\sigma)}\|x(s)-y(s)\| .
\end{aligned}
$$

Expression (3.36) together with (3.37) imply that $\mathcal{F}_{n}^{\tau}$ is a contraction. By the Banach contraction principle, it results that for each $\tau \in[0,1)$, the equation $\left.x\right|_{[\sigma, \tau T+\sigma+h]}=\left.\mathcal{F}_{n}^{\tau}(x)\right|_{[\sigma, \tau T+\sigma+h]}$ has (a unique) solution in $\left.D\left(x_{n}, \varphi\right)\right|_{[\sigma, \tau T+\sigma+h]}$. Thus, problem (3.24) has a local solution defined on $[\sigma, \tau T+\sigma+h]$ for some $h>0$.

Denote by $\bar{z}_{n}^{\tau}$ that solution. Since $\widehat{f}$ instead of $F_{n}$ satisfies conditions $\left(\mathrm{F}_{1}\right)$, $\left(\mathrm{F}_{2}\right),\left(\mathrm{F}_{3}^{\prime}\right)$ and $\left(\mathrm{F}_{4}\right)$, by hypothesis $\left(\mathcal{I}_{2}\right)$, the function $\bar{z}_{n}^{\tau}$ has an extension to all of $[\sigma, T+\sigma]$. Lemma 3.19 ensures that this extension belongs to $\Sigma_{\varphi(0)}^{F_{n}}$. Let $z_{n}^{\tau} \in \Sigma_{\varphi(0)}^{F_{n}}$ be an extension of $\bar{z}_{n}^{\tau}$ to all of $[\sigma, T+\sigma]$.

LEMMA 3.22. $z_{n}^{\tau}$ is unique.

ProOF. Let $z \in \Sigma_{\varphi(0)}^{F_{n}}$ be another extension of $\bar{z}_{n}^{\tau}$ to all of $[\sigma, T+\sigma]$. Then $z_{n}^{\tau}(t)=z(t)$ for all $t \in[\sigma, \tau T+\sigma+h]$, where $h>0$. Set

$$
\mathcal{A}=\left\{t \in[\sigma, T+\sigma]: z_{n}^{\tau}(s)=z(s) \text { for each } s \in[\sigma, t]\right\} .
$$

As $z_{n}^{\tau}$ and $z$ are continuous and $z_{n}^{\tau}(s)=z(s)$ for each $t \in[\sigma, \tau T+\sigma+h]$, the set $\mathcal{A}$ is nonempty bounded and closed, hence compact. Set $a=\max \mathcal{A}$. It is clear that $\sigma+\tau T+h \leq a \leq T+\sigma$. Let us prove that $a=\sigma+T$. Assume the contrary. Then for each $t \in[\sigma, a]$ with $a<T+\sigma$, we have $z_{n}^{\tau}(s)=z(s)$. Reasoning as above, we can see that $z_{n}^{\tau}$ and $z$ must coincide on an interval of the form $\left[\sigma, a+h^{\prime}\right]$, with $h^{\prime}>0$ sufficiently small. Since this statement contradicts the definition of $a$, it follows that the supposition $a<T+\sigma$ is false. Then $a=T+\sigma$.

Proof of Lemma 3.16. Let $n \geq 1$ be fixed. Let us define the deformation $h:[0,1] \times \Sigma_{\varphi(0)}^{F_{n}} \rightarrow \Sigma_{\varphi(0)}^{F_{n}}$ by

$$
h\left(\tau, x_{n}\right)= \begin{cases}z_{n}^{\tau} & \text { for } 0 \leq \tau<1 \\ x_{n} & \text { for } \tau=1\end{cases}
$$

It is clear that $h(0, \cdot)=z_{n}^{0}$ and $h(1, \cdot)$ is the identity. 
It remains to prove that $h$ is continuous. Since, for all $t \in[\sigma, T+\sigma], z_{n}^{\tau}(t)=$ $S\left(\mathbf{1}_{[\sigma, \tau T+\sigma]} f+\mathbf{1}_{[\sigma+\tau T, T+\sigma]} G_{n}^{\tau}\left(z_{n}^{\tau}\right)\right)(t)$, where $f \in \overline{\operatorname{sel}}_{F}(x)$ is such that $x_{n}(\cdot)=$ $S(f)(\cdot)$, we have

$$
h\left(\tau, x_{n}\right)= \begin{cases}S\left(\mathbf{1}_{[\sigma, \tau T+\sigma]} f+\mathbf{1}_{[\sigma+\tau T, T+\sigma]} G_{n}^{\tau}\left(z_{n}^{\tau}\right)\right) & \text { for } 0 \leq \tau<1, \\ x_{n} & \text { for } \tau=1 .\end{cases}
$$

Recall that by property $\left(2 \mathrm{~S}_{1}\right)$ (see Lemma 3.7 ) of the operator $S$, the value $h\left(\tau, x_{n}\right)$ does not depend on the choice of $f$. As

$$
\left(\mathbf{1}_{[\sigma, T+\sigma]} f+\mathbf{1}_{\{\sigma+T\}} G_{n}^{\tau}\left(z_{n}^{\tau}\right)\right)(t)=f(t), \quad \text { for a.e. } t \in[\sigma, T+\sigma],
$$

we have

$$
x_{n}=S(f)=S\left(\mathbf{1}_{[\sigma, T+\sigma]} f+\mathbf{1}_{[\sigma+T, T+\sigma]} G_{n}^{\tau}\left(z_{n}^{\tau}\right)\right)=h\left(1, x_{n}\right) .
$$

Hence, for all $\left(\tau, x_{n}\right) \in[0,1] \times \Sigma_{\varphi(0)}^{F_{n}}$, one can write

$$
h\left(\tau, x_{n}\right)=S\left(\mathbf{1}_{[\sigma, \tau T+\sigma]} f+\mathbf{1}_{[\sigma+\tau T, T+\sigma]} G_{n}^{\tau}\left(z_{n}^{\tau}\right)\right) .
$$

It is clear that by condition $\left(1 \mathrm{~S}_{1}\right)$ (see Lemma 3.7 ), we have

$$
h\left(\tau, x_{n}\right)(t)=S(f)(t)=x_{n}(t), \quad \text { for all } t \in[\sigma, \tau T+\sigma] .
$$

Let $\left(\tau_{k}, x_{k}\right) \in[0,1] \times \Sigma_{\varphi(0)}^{F_{n}}$ with $\left(\tau_{k}, x_{k}\right) \stackrel{k \rightarrow \infty}{\longrightarrow}(\tau, x)$. Set

$$
\psi_{k}(t)=\left\|h(\tau, x)(t)-h\left(\tau_{k}, x_{k}\right)(t)\right\| .
$$

We are going to prove that $\sup _{t \in[\sigma, T+\sigma]} \psi_{k}(t) \stackrel{k \rightarrow \infty}{\longrightarrow} 0$. We consider only the case $\tau_{k} \leq \tau$, the remaining cases can be treated similarly. For $\varepsilon>0$, let $\delta>0$ be chosen such that for every measurable set $I \subset[\sigma, T+\sigma]$, meas $(I) \leq \delta$, we have

$$
\int_{I} \bar{\alpha}(s) d s<\frac{\varepsilon}{M},
$$

where $\bar{\alpha}$ is from the estimate $\left(\mathrm{F}_{3}^{\prime}\right)$ and $M$ is from $\left(\mathrm{S}_{1}\right)$. Choose $k_{\varepsilon} \geq 1$ so that, for all $k \geq k_{\varepsilon}$,

$$
\left\|x_{k}-x\right\|_{C([\sigma, T+\sigma] ; E)} \leq \frac{\varepsilon}{M}, \quad\left|\tau_{k}-\tau\right| \leq \frac{\delta}{T} .
$$

Let $k \geq k_{\varepsilon}$. We distinguish three cases:

Case 1. If $t \in\left[\sigma, \tau_{k} T+\sigma\right]$, then by (3.38), we get

$$
\psi_{k}(t)=\left\|x(t)-x_{k}(t)\right\| \leq \frac{\varepsilon}{M} .
$$

Case 2. If $t \in\left[\sigma+\tau_{k} T, \tau T+\sigma\right]$, then using condition $\left(\mathrm{S}_{1}\right),(3.39)$ and (3.40), we obtain

$$
\begin{aligned}
\psi_{k}(t) & =\left\|S(f)(t)-S\left(\mathbf{1}_{\left[\sigma, \tau_{k} T+\sigma\right]} f_{k}+\mathbf{1}_{\left[\tau_{k} T+\sigma, T+\sigma\right]} G_{n}^{\tau}\left(z_{k}^{\tau_{k}}\right)\right)\right\| \\
& \leq M\left\|S(f)\left(\sigma+\tau_{k} T\right)-S\left(\mathbf{1}_{\left[\sigma, \tau_{k} T+\sigma\right]} f_{k}+\mathbf{1}_{\left[\tau_{k} T+\sigma, T+\sigma\right]} G_{n}^{\tau}\left(z_{k}^{\tau_{k}}\right)\right)\left(\sigma+\tau_{k} T\right)\right\|
\end{aligned}
$$




$$
\begin{aligned}
& +M \int_{\sigma+\tau_{k} T}^{t}\left\|f(s)-g\left(s, z_{n}^{\tau_{k}}[\varphi]_{s}\right)\right\| d s \\
\leq & M\left\|x\left(\sigma+\tau_{k} T\right)-x_{k}\left(\sigma+\tau_{k} T\right)\right\|+2 M \int_{\tau_{k} T+\sigma}^{\tau T+\sigma}\|\bar{\alpha}(s)\| d s \\
\leq & M \frac{\epsilon}{M}+2 M \frac{\epsilon}{M}=3 \epsilon .
\end{aligned}
$$

Set $\mathcal{K}=\left\{x[\varphi]_{t}: t \in[\sigma, t+\sigma], x \in \Sigma_{\varphi(0)}^{F_{n}}\right\}$. From Corollary 3.11, we know that $\Sigma_{\varphi(0)}^{F_{n}}$ is compact. Taking $\Sigma_{\varphi(0)}^{F_{n}}$ instead of $X$ in Corollary 3.13, we deduce that $\mathcal{K}$ is compact. Let $c_{1}(\mathcal{K}), c_{2}(\mathcal{K})>0$ be such that (3.18) holds with $\mathfrak{K}=\mathcal{K}$. We have for all $k \geq k_{\varepsilon}$,

Case 3. If $t \in[\sigma+\tau T, T+\sigma]$, then, by means of condition $\left(\mathrm{S}_{1}\right)$, Lemma $3.15(\mathrm{~d})$, and the last inequality in Case 2, we obtain

$$
\begin{aligned}
\psi_{k}(t) \leq & M \| S\left(\mathbf{1}_{[\sigma, \tau T+\sigma]} f+\mathbf{1}_{[\tau T+\sigma, T+\sigma]} G_{n}^{\tau}\left(z^{\tau}\right)\right)(\sigma+\tau T) \\
& -S\left(\mathbf{1}_{\left[\sigma, \tau_{k} T+\sigma\right]} f_{k}+\mathbf{1}_{\left[\tau_{k} T+\sigma, T+\sigma\right]} G_{n}^{\tau}\left(z_{k}^{\tau_{k}}\right)\right)(\sigma+\tau T) \| \\
& \left.+M \int_{\sigma+\tau T}^{t} \| g\left(s, z^{\tau}[\varphi]_{s}\right)-g\left(s, z_{k}^{\tau_{k}}[\varphi]_{s}\right)\right) \| d s \\
\leq & \psi_{k}(\sigma+\tau T)+M \int_{\tau T+\sigma}^{t}\left\|g\left(s, z^{\tau}[\varphi]_{s}\right)-g\left(s, z_{k}^{\tau_{k}}[\varphi]_{s}\right)\right\| d s \\
\leq & 3 M \varepsilon+M c_{1}(\mathcal{K}) \int_{\sigma+\tau T}^{t} \bar{\alpha}(\gamma)\left\|z^{\tau}[\varphi]_{\gamma}-z_{k}^{\tau_{k}}[\varphi]_{\gamma}\right\|_{\mathcal{B}} d \gamma \\
\leq & 3 M \varepsilon+M c_{1}(\mathcal{K}) \int_{\sigma+\tau T}^{t} \bar{\alpha}(\gamma)\left[\beta_{K} \sup _{\sigma \leq s \leq \gamma}\left\|z^{\tau}(s)-z_{k}^{\tau_{k}}(s)\right\|\right. \\
& \left.+\beta_{N}\left\|z^{\tau}[\varphi]_{\sigma}-z_{k}^{\tau_{k}}[\varphi]_{\sigma}\right\|\right] d \gamma \\
\leq & \left.3 M \varepsilon+M c_{1}(\mathcal{K}) \int_{\sigma+\tau T}^{t} \bar{\alpha}(\gamma)\left[\beta_{K} \sup _{\sigma \leq s \leq \gamma}\left\|z^{\tau}(s)-z_{k}^{\tau_{k}}(s)\right\|+\beta_{N} \| \varphi-\varphi\right] \|_{\mathcal{B}}\right] d \gamma \\
\leq & 3 M \varepsilon+M \beta_{K} c_{1}(\mathcal{K}) \int_{\sigma+\tau T}^{t} \bar{\alpha}(\gamma)\left[\sup _{\sigma \leq s \leq \sigma+\tau T}\left\|z^{\tau}(s)-z_{k}^{\tau_{k}}(s)\right\|\right. \\
& \left.+\sup _{\sigma+\tau T \leq s \leq \gamma}\left\|z^{\tau}(s)-z_{k}^{\tau_{k}}(s)\right\|\right] d \gamma \\
\leq & 3 M \varepsilon+M \beta_{K} c_{1}(\mathcal{K}) \int_{\sigma+\tau T}^{t} \bar{\alpha}(\gamma)\left[3 \varepsilon+\sup _{\sigma+\tau T \leq s \leq \gamma}\left\|z^{\tau}(s)-z_{k}^{\tau_{k}}(s)\right\|\right] d \gamma \\
\leq & 3 M \varepsilon+3 \varepsilon M \beta_{K} c_{1}(\mathcal{K}) \int_{\sigma+\tau T}^{t} \bar{\alpha}(\gamma) d \gamma \\
& +M \beta_{K} c_{1}(\mathcal{K}) \int_{\sigma+\tau T}^{t} \bar{\alpha}(\gamma) \sup _{\sigma+\tau T \leq s \leq \gamma}\left\|z^{\tau}(s)-z_{k}^{\tau_{k}}(s)\right\| d \gamma \\
& \\
&
\end{aligned}
$$




$$
\leq 3\left(M+M \beta_{K} c_{1}(\mathcal{K})\|\bar{\alpha}\|_{L^{1}}\right) \varepsilon+M \beta_{K} c_{1}(\mathcal{K}) \int_{\sigma+\tau T}^{t} \bar{\alpha}(\gamma) \sup _{\sigma+\tau T \leq s \leq \gamma} \psi(s) d \gamma,
$$

where $\beta_{K}$ from (3.10) and $\beta_{N}$ form (3.11). Since the last expression does not decrease, we have

$$
\begin{aligned}
\sup _{\sigma+\tau T \leq s \leq t} \psi(s) \leq 3\left(1+M \beta_{K} c_{1}(\mathcal{K})\|\bar{\alpha}\|_{L^{1}}\right) \varepsilon & \\
& +M \beta_{K} c_{1}(\mathcal{K}) \int_{\sigma+\tau T}^{t} \bar{\alpha}(\gamma) \sup _{\sigma+\tau T \leq s \leq \gamma} \psi(s) d \gamma .
\end{aligned}
$$

Thus, applying the Gronwall-Bellmann inequality to the function

$$
\widehat{\psi}(t)=\sup _{\sigma+\tau T \leq s \leq t} \psi(s),
$$

we obtain

$$
\widehat{\psi}(t) \leq 3\left(1+M \beta_{K} c_{1}(\mathcal{K})\|\bar{\alpha}\|_{L^{1}}\right) \varepsilon \exp \left(M \beta_{K} c_{1}(\mathcal{K}) \int_{\sigma+\tau T}^{T+\sigma} \bar{\alpha}(\gamma) d \gamma\right) .
$$

The continuity of $h$ follows from the arbitrariness of $\varepsilon$. This achieves the proof of Lemma 3.16.

In the proof of our main result we will need the following simple lemma.

Lemma 3.23. Let $E_{1}, E_{2}$ be two metric spaces and let $\varphi: E_{1} \rightarrow E_{2}$ be a continuous injective application. If $C$ is an $R_{\delta}$-set in $E_{1}$, then $\varphi(C)$ is an $R_{\delta}$-set in $E_{2}$.

Proof. Let $C$ be an $R_{\delta}$-set in $E_{1}$ with $C=\bigcap_{n \geq 1} C_{n}, C_{n}$ compact contractible and, $C_{n+1} \subset C_{n}$, for all $n \geq 1$. It is clear that $\varphi\left(C_{n}\right)$ is a compact set and $\varphi\left(C_{n+1}\right) \subset \varphi\left(C_{n}\right)$ for all $n \geq 1$. For any $n \geq 1$, let $h_{n}:[0,1] \times C_{n} \rightarrow C_{n}$ be a continuous function such that, for every $x \in C_{n}, h_{n}(0, x)=x_{0}^{n} \in C_{n}$ and $h_{n}(1, x)=x$. Let $\widetilde{h}_{n}:[0,1] \times \varphi\left(C_{n}\right) \rightarrow \varphi\left(C_{n}\right)$ be defined by

$$
\widetilde{h}_{n}(\tau, y)=\varphi\left(h_{n}(\tau, x)\right),
$$

where $x \in C_{n}$ is the unique element (since $\varphi$ is injective) such that $y=\varphi(x)$. It is clear that $\widetilde{h}_{n}(\cdot, \cdot), n \geq 1$, is continuous and satisfies for every $y \in \varphi\left(C_{n}\right)$,

$$
\begin{aligned}
& \widetilde{h}_{n}(0, y)=\varphi\left(h_{n}(0, x)\right)=\varphi\left(x_{0}^{n}\right) \in \varphi\left(C_{n}\right), \\
& \widetilde{h}_{n}(1, y)=\varphi\left(h_{n}(1, x)\right)=\varphi(x)=y .
\end{aligned}
$$

Thus for all $n \geq 1, \varphi\left(C_{n}\right)$ is contractible. Using again the fact that $\varphi$ is injective, we have

$$
\varphi(C)=\varphi\left(\bigcap_{n \geq 1} C_{n}\right)=\bigcap_{n \geq 1} \varphi\left(C_{n}\right) .
$$

Therefore $\varphi(C)$ is an $R_{\delta}$-set. 
Proof of Main Result (Proof of Theorem 3.1). Let us prove that

$$
\Sigma_{\varphi(0)}^{\widehat{F}}=\bigcap_{n \geq 1} \Sigma_{\varphi(0)}^{F_{n}},
$$

where $\widehat{F}$ is given by (3.17). From Lemma $3.15(\mathrm{~b})$, we immediately have that

$$
\Sigma_{\varphi(0)}^{\widehat{F}} \subset \bigcap_{n \geq 1} \Sigma_{\varphi(0)}^{F_{n}} .
$$

Let us prove the converse inclusion. Let $x \in \bigcap_{n \geq 1} \Sigma_{\varphi(0)}^{F_{n}}$. Then, for each $n \geq 1$, there exists a function $f_{n}$ such that

$$
f_{n} \in L^{1}([\sigma, T+\sigma] ; E), \quad f_{n}(t) \in F_{n}\left(t, x[\varphi]_{t}\right), \quad \text { for a.e. } t \in[\sigma, T+\sigma],
$$

and $x(\cdot)=S\left(f_{n}\right)(\cdot)$. From Lemma $3.15(\mathrm{~b})$, we have, for all $n \geq 1$

$$
F_{n}\left(t, x[\varphi]_{t}\right) \subseteq \overline{\operatorname{co}} F\left(t, X[\varphi]_{t}\right), \quad \text { for a.e. } t \in[\sigma, T+\sigma]
$$

where $X$ is from Lemma 3.10. Hence using condition $\left(\mathrm{F}_{4}\right)$, we obtain

$$
\chi\left(\left\{f_{n}(t)\right\}_{n=1}^{\infty}\right) \leq \chi\left(\overline{\operatorname{co}} F\left(t, X[\varphi]_{t}\right)\right)=\chi\left(F\left(t, X[\varphi]_{t}\right)=0,\right.
$$

for almost every $t \in[\sigma, T+\sigma]$. Further, condition $\left(\mathrm{F}_{3}^{\prime}\right)$ ensures that the sequence $\left\{f_{n}\right\}_{n=1}^{\infty}$ is integrably bounded. Consequently, the sequence $\left\{f_{n}\right\}_{n=1}^{\infty}$ is semicompact, so we can assume without loss of generality that

$$
f_{n} \stackrel{\mathrm{w}}{\longrightarrow} f_{0} \in L^{1}([\sigma, T+\sigma] ; E) \quad \text { when } n \rightarrow \infty .
$$

Applying Lemma 3.4, we have, for almost every $t \in[\sigma, T+\sigma]$ and for all $n \geq 1$,

$$
f_{0}(t) \in F_{n}\left(t, x[\varphi]_{t}\right), \quad \text { for a.e. } t \in[\sigma, T+\sigma] .
$$

Hence, using Lemma 3.15 (c), we obtain, for almost every $t \in[\sigma, T+\sigma]$,

$$
f_{0}(t) \in \bigcap_{n \geq 1} F_{n}\left(t, x[\varphi]_{t}\right)=\widehat{F}\left(t, x[\varphi]_{t}\right) .
$$

By Lemma 3.8, we have $S\left(f_{n}\right) \rightarrow S\left(f_{0}\right)$ when $n \rightarrow \infty$. But for all $n \geq 1$, $S\left(f_{n}\right)(\cdot)=x(\cdot)$, then $S\left(f_{0}\right)(\cdot)=x(\cdot)$. Consequently, $x \in \Sigma_{\varphi(0)}^{\widehat{F}}$. Thus

$$
\Sigma_{\varphi(0)}^{\widehat{F}}=\bigcap_{n \geq 1} \Sigma_{\varphi(0)}^{F_{n}} .
$$

From Lemma $3.15(\mathrm{~b})$, we have $\Sigma_{\varphi(0)}^{F_{n+1}} \subset \Sigma_{\varphi(0)}^{F_{n}}, n \geq 1$. Hence invoking Lemma 3.16, we deduce that $\Sigma_{\varphi(0)}^{\widehat{F}}$ is an $R_{\delta}$-set. Corollary 3.11 and Lemma 3.14 imply that $\Sigma_{\varphi(0)}^{F}$ is a compact $R_{\delta}$-set.

The application $\Phi: D(\varphi, T) \rightarrow C((-\infty, T+\sigma] ; E)$ defined by $\Phi(x)=x[\varphi]$ is continuous and injective. Applying Lemma 3.23, we conclude that the set $\Sigma_{\varphi}^{F}=\Phi\left(\Sigma_{\varphi(0)}^{F}\right)$ is a compact $R_{\delta}$-set in $C((-\infty, T+\sigma] ; E)$. 


\section{Applications}

Let $F:[\sigma, T+\sigma] \times \mathcal{B} \rightarrow K v(E)$ be a multivalued map satisfying conditions $\left(\mathrm{F}_{1}\right)-\left(\mathrm{F}_{4}\right)$ and let $\varphi \in \mathcal{B}$ be a fixed element, where $\mathcal{B}$ is a Banach space of functions mapping $(-\infty ; 0]$ into $E$ satisfying conditions (B1)-(B4). Consider the quasi-linear problem

$$
\left\{\begin{array}{l}
y^{\prime}(t) \in A(t, y(t))+F\left(t, y_{t}\right) \quad \text { for } t \in[\sigma, T+\sigma], \\
y_{\sigma}=\varphi
\end{array}\right.
$$

As an operator $S: L^{1}([\sigma, T+\sigma] ; E) \rightarrow D(\varphi, T)$, we take the solution operator of the quasi-linear problem

$$
\left\{\begin{array}{l}
y^{\prime}(t) \in A(t, y(t))+f(t) \quad \text { for } t \in[\sigma, T+\sigma], \\
y(\sigma)=\varphi(0),
\end{array}\right.
$$

i.e. for each $f \in L^{1}([\sigma, T+\sigma] ; E)$, the value $S(f)$ stands for the (unique) solution of (4.2). Then, problem (4.1) can be written as

$$
\left\{\begin{array}{l}
y \in S \circ \operatorname{sel}_{F}(y) \\
y_{\sigma}=\varphi
\end{array}\right.
$$

Let us describe some concrete situations.

Semilinear case. $A(t, x(t))=A x(t)$, where $A: D(A) \subset E \rightarrow E$ a linear not necessarily bounded operator generating a strongly $C_{0}$-semigroup $\left\{e^{A t}, t \geq 0\right\}$. By means of the variation of constants formula, the mild solution operator $S$ associated with the inclusion (4.1) can be expressed explicitly by

$$
\begin{gathered}
S: L^{1}([\sigma, T+\sigma] ; E) \rightarrow C([\sigma, T+\sigma] ; E), \\
S(f)(t)=e^{-A(t-\sigma)} \varphi(0)+\int_{\sigma}^{t} e^{-A(t-s)} f(s) d s .
\end{gathered}
$$

Let $M>0$ be a constant such that $\left\|e^{-A t}\right\| \leq M$, for all $t \in[\sigma, T+\sigma]$. It is easy to see that $S$ satisfies condition $\left(\mathrm{S}_{1}\right)$. Condition $\left(\mathrm{S}_{2}\right)$ follows from [25, 4.2.1]. According to [18, Theorems 2, 3], the solution set $\Sigma_{\varphi}^{F}$ is nonempty and compact (hence bounded) and each local solution has an extension to all $(-\infty, T+\sigma]$. Thus, the solution set of (4.1) is a compact $R_{\delta}$-set in $\mathcal{C}((-\infty, T+\sigma] ; E)$.

Fully nonlinear case. $A(t, x(t))=-A x(t)$, where $A: D(A) \subset E \rightarrow \mathcal{P}(E)$ is an $m$-accretive operator, with $0 \in A 0$, such that $-A$ generates an equicontinuous semigroup not necessarily compact. Suppose that $\varphi(0) \in \overline{D(A)}$. Let $S: L^{1}([\sigma, T+\sigma] ; E) \rightarrow C([\sigma, T+\sigma] ; E)$ be the integral solution operator, i.e. for every $f \in L^{1}([\sigma, T+\sigma] ; E)$, the value $S(f)$ is the unique integral solution of problem (4.2). Suppose that the dual space $E^{*}$ of $E$ is uniformly convex, then one can invoke [5, Proposition 1, Lemma 4] to infer that the operator $S$ satisfy $\left(\mathrm{S}_{2}\right)$. 
Condition $\left(\mathrm{S}_{1}\right)$ follows from the fact that if $y_{i}, i=1,2$, is the integral solution to the problem

$$
\left\{\begin{array}{l}
y_{i}^{\prime}(t) \in-A y_{i}(t)+g_{i}(t) \quad \text { for } a<t \leq b \\
y_{i}(a)=y_{i}^{0} \in \overline{D(A)}
\end{array}\right.
$$

then

$$
\left\|y_{1}(t)-y_{2}(t)\right\| \leq\left\|y_{1}(s)-y_{2}(s)\right\|+\int_{s}^{t}\left\|g_{1}(\tau)-g_{2}(\tau)\right\| d \tau,
$$

for all $a \leq s \leq t \leq b$. (For more details see [4].) According to [18, Theorems 4.1, 5.1], the solution set $\Sigma_{\varphi}^{F}$ is nonempty and compact (hence bounded) and each local solution has an extension to all $(-\infty, T+\sigma]$. From Theorem 3.1, we deduce that $\Sigma_{\varphi}^{F}$ is a compact $R_{\delta}$-set in $\mathcal{C}((-\infty, T+\sigma] ; E)$.

Nonautonomous semilinear case. $A(t, x(t))=A(t) x(t)$, where family $\{A(t)\}_{t \in[0, T]}$ is a family of closed, not necessarily bounded operators in $E$ generating a strongly continuous evolution operator $U: \Delta \rightarrow \mathcal{L}(E)$, where $\Delta=\{t, s \in$ $[0, T]: s \leq t\}$ and $\mathcal{L}(E)$ is the space of all bounded linear operators in $E$.

$\left(\mathrm{U}_{1}\right)$ For any $x \in E$, the function $(t, s) \mapsto U(t, s) x$ is continuous in $\Delta$;

$\left(\mathrm{U}_{2}\right)$ for all $(t, s),(s, \theta) \in \Delta$, the relations $U(t, s) \circ U(s, \theta)$ and $U(t, t)=I_{E}$.

The mild solution operator $S$ associated with inclusion (4.1), with $\sigma=0$, can be expressed explicitly by

$$
\begin{gathered}
S: L^{1}([0, T] ; E) \rightarrow C([0, T] ; E), \\
S(f)(t)=U(t, 0) \varphi(0)+\int_{0}^{t} U(t, s) f(s) d s .
\end{gathered}
$$

Let $M>0$ be a constant such that $\|U(t, s)\|_{\mathcal{L}(E)} \leq M$, for all $(t, s) \in \Delta$. It is clear that $S$ satisfies condition $\left(\mathrm{S}_{1}\right)$. Condition $\left(S_{2}\right)$ follows from [7, Theorem 2] (see also [11]). If conditions $\left(\mathcal{I}_{1}\right)$ and $\left(\mathcal{I}_{2}\right)$ of Theorem 3.1 are satisfied, then $\Sigma_{\varphi}^{F}$ is a compact $R_{\delta}$-set in $\mathcal{C}((-\infty, T] ; E)$.

REMARK 4.1. (a) In our approach, the space $\mathcal{B}$ was considered as a Banach space, however it is more natural to consider it as a seminormed space. Another approach would be to assume that $\mathcal{B}$ is a complete seminormed space satisfying axioms (B1)-(B4) and to show that the solutions observed only for $t \in[\sigma, T+\sigma]$ form a compact $R_{\delta}$-subset in $C([\sigma, T+\sigma] ; E)$. In this case, the space $\mathcal{C}((-\infty$, $\sigma+T] ; E$ ) becomes a complete seminormed space and many of our preliminary results are valid, but the major constraint is Lemma 3.15 that played a key role in the proof of our main result. This is why we choose $\mathcal{B}$ as a Banach space.

(b) In Section 4 where some concrete situations are described, it is not assumed that the operator $A$ generates necessarily a compact semigroup. This is one of the interesting points of this work. 


\section{REFERENCES}

[1] R.R. Akhmerov, M.I. Kamenskĭ, A.S. Potapov, B.N. Rodkina and B.N. Sadovskĭ, Measures of Noncompactness and Condensing Operators, Birkhäuser, 1992.

[2] N. Aronszajn, Le correspondant topologique de l'unicité dans la théorie des équations différentielles, Ann Math. 43 (1942), 730-738.

[3] J. Banas and K. Goebel, Measures of Noncompactness in Banach Spaces, Marcel Dekker, New York, 1980.

[4] V. BARBu, Nonlinear Semigroups and Differential Equations in Banach Spaces, Editura Academiei Bucharest-Noordhoff, Leyden, 1976.

[5] D. Bотне, Multivalued perturbations of $m$-accretive differential inclusions, Israel J. Math. 108 (1998), 109-138.

[6] H. BrezIS, Functional Analysis, Sobolev Spaces and Partial Differential Equations, Springer Science \& Business Media, 2010.

[7] T. Cardinali and P. RubBioni, On the existence of mild solutions of semilinear evolution differential inclusions, J. Math. Anal. Appl. 308 (2005), 620-635.

[8] M. Cichoń And I. KubiaczyK, Some remarks on the structure of the solution set for differential inclusions in Banach spaces, J. Math. Anal. Appl. 233 (1999), 597-606.

[9] B.D. Coleman And D.R. Owen, On the initial value problem for a class of functionaldifferential equations, Arch. Ration. Mech. Anal. 55 (1974), 275-299.

[10] G. Conti, V.V. Obukhovskĭ And P. ZECCA, On the topological structure of the solution set for a semilinear functional-differential inclusion in a Banach space, Banach Center Publications 35 (1996), 159-169.

[11] J.F. Couchouron And M. Kamenskil̆, A unified topological point of view for integrodifferential inclusions, Lecture Notes in Nonlinear Anal. 2 (1998), 123-137.

[12] F.S. De Blasi And J. MyjaK, On the solution sets for differential inclusions, Bull. Polon. Acad. Sci. 33 (1985), 17-23.

[13] K. Deimling, Multivalued Differential Equations, de Gruyter, Berlin, 1992.

[14] K. Deimling and M.R. Mohana RaO, On solution sets of multivalued differential equations, Appl. Anal. 30 (1988), 129-135.

[15] J. Diestel, W.M. Ruess and W. Schachermayer, On weak compactness in $L^{1}(\mu, X)$, Proc. Amer. Math. Soc. 118 (1993), 447-453.

[16] S. Djebali, L. Górniewicz and A. Ouahab, Solution sets for differential equations and inclusions, Walter de Gruyter, 2012.

[17] C. Gori, V. Obukhovskĭ, M. Ragni and P. Rubbioni, On some properties of semilinear functional differential inclusions in abstract spaces, J. Concr. Appl. Math. 4 (2006), no. 2, 183-214.

[18] C. Gori, V.V. Obukhovskiı̆, M. Ragni and P. Rubbioni, Existence and continuous dependence results for semilinear functional differential inclusions with infinite delay, Nonlinear Anal. 51 (2002), 765-782.

[19] L. Gónniewicz, Topological fixed point theory of multivalued mappings, Springer, 2006 (second edition)

[20] _ Topological Structure of Solution Sets: Current Results, Arch. Math. (Brno) 36 (2000), 343-382.

[21] J.K. Hale And J. Kato, Phase space for retarded equations with infinite delay, Funkcial. Ekvac. 21 (1978), 11-41.

[22] C.J. Himmelberg and F.S. Van Vleck, A note on the solution sets of differential inclusions, Rocky Mountain J. Math. 12 (1982), 621-625. 
[23] Y. Hino, S. Murakami and T. Naito, Functional-differential equations with infinite delay, Springer-Verlag, 1991.

[24] Y. Hino, T. Naito, N.V. Minh And J.S. Shin, Almost periodic solutions of differential equations in Banach spaces, Taylor \& Francis, 2002.

[25] M.I. KamenskiĬ, V.V. ObukhovskiI and P. ZecCA, Condensing Multivalued Maps and Semilinear Differential Inclusions in Banach Spaces, Walter de Gruyter \& Co., 2001.

[26] F. Kappel and W. Schappacher, Some considerations to the fundamental theory of infinite delay equations, J. Differential Equations. 37 (1980), 141-183.

[27] N.S. Papageorgiou, On the solution set of differential inclusions in Banach space, Appl. Anal. 25 (1987), 319-329.

[28] K. Schumacher, Existence and continuous dependence for functional-differential equations with unbounded delay, Arch. Rational Mech. Anal. 67 (1978), 315-335.

Manuscript received June 16, 2015

accepted December 13, 2015

LAHCENE GUEDDA

Laboratoire de Génie Électrique et Génie Informatique

Université de Tiaret

BP 78. Zaaroura

14000 Tiaret, ALGÉRIE

E-mail address: lahcene_guedda@yahoo.fr 